\title{
A New Approach to Weapon-Target Assignment in Cooperative Air Combat
}

\author{
Yi-zhe Chang, ${ }^{1}$ Zhan-wu Li, ${ }^{1}$ Ying-xin Kou, ${ }^{1}$ Qing-peng Sun, \\ Hai-yan Yang, ${ }^{1}$ and Zheng-yan Zhao ${ }^{2}$ \\ ${ }^{1}$ Air Force Engineering University, Xian 710038, China \\ ${ }^{2} 93088$ Unit of PLA, Chifeng 024400, China \\ Correspondence should be addressed to Yi-zhe Chang; 272884459@qq.com
}

Received 9 May 2017; Accepted 8 August 2017; Published 2 October 2017

Academic Editor: Jean Jacques Loiseau

Copyright (C) 2017 Yi-zhe Chang et al. This is an open access article distributed under the Creative Commons Attribution License, which permits unrestricted use, distribution, and reproduction in any medium, provided the original work is properly cited.

A new approach to solving weapon-target assignment (WTA) problem is proposed in this paper. Firstly, relative superiority that lays the foundation for assignment is calculated based on the combat power energy of the fighters. Based on the relative superiority, WTA problem is formulated. Afterwards, a hybrid algorithm consisting of improved artificial fish swarm algorithm (AFSA) and improved harmony search (HS) is introduced and furthermore applied to solve the assignment formulation. Finally, the proposed approach is validated by eight representative benchmark functions and two concrete cooperative air combat examples. The results show that the approach proposed in this paper achieves good performances in solving WTA problem in cooperative air combat.

\section{Introduction}

Weapon-target assignment (WTA) refers to an assignment of defensive weapons to engage or counter identified threats. The primary concern is minimizing the total expected survivability of the targets $[1,2]$. Weapons are assigned to threats based on the detection outcomes within previous stages. Considering the short time of air combat, the problem must be solved as close to real time as possible. Another characteristic is quality of the derived solution, which causes critical effects on the following combat deployment [3].

WTA is regarded as a crucial part in operation; therefore the achievements on that are rich. In the matter of problem formulation, multiple factors such as relative distance, relative angle, and relative velocity are taken into consideration. In domain of algorithms, several sophisticated search and heuristic algorithms have been proposed, such as genetic algorithms [4-7], simulated annealing [8], discrete particle swarm optimizations [9-12], permutation and tabu search heuristics [13], and other algorithms [14-16].

Concerned with the characteristics and developing tendency about air combat, an approach to WTA in cooperative air combat is proposed in this paper. Within the approach, relative superiority between fighters and targets, acting as the foundation of the formulation, is calculated in terms of combat power potential, and a hybrid algorithm combined with improved artificial fish swarm algorithm with improved harmony search for solving the formulation is introduced.

The concept of combat power potential is firstly introduced by Zhou et al. [17]. As described in [17], air combat space can be seen as a potential consisting of infinite points and each point is under effect by fighters. The effect, generated by fighters and equipment carried by them, is quantitatively defined as potential energy called combat power potential energy. Combat power potential energy reflects the impact on each point inside the air combat space by fighters.

The artificial fish swarm algorithm (AFSA) simulating the behavior of a fish inside water was proposed by Li et al. [18] and has already been applied in engineering contexts [1924]. The main artificial fish behaviors are random, chasing, swarming, and searching, which allows the algorithm to act better in global searching but poor in local searching.

Harmony search, mimicking the improvisation process of music players, is originated by Geem et al. [25]. The HS performs excellently in quality of the solutions but with low search speed and is strongly dependent on initial solution. 
This paper is organized as follows: In Section 2, the formulation of WTA is introduced. The design of hybrid algorithm is studied in Section 3. Section 4 presents the results of simulation. Section 5 concludes the paperpagebreak

\section{WTA Problem Formulation}

2.1. Analysis on Relative Superiority. Superiority relation acts as the critical ingredient in WTA formulation. In this paper, superiority relation is formulated in accordance with the descending velocity of combat power potential energy possessed by one side towards the other side.

2.1.1. Combat Power Potential Energy Model of Fighter. Due to the limitation on pages, combat power potential energy of fighter can be calculated by model of which in [26].

2.1.2. Calculation on Descending Velocity. We get to know from the potential theory that potential energy will be descending while distance is getting larger. As a kind of potential, combat power potential has the same characteristics, which means combat power potential energy generated by fighters descends with more restriction, such as distance limits and angle limits. For calculating descending velocity, gradient is introduced in the paper. Assuming a function described as $f=f(P), P=(x, y, z)$, then gradient of function $f$ in point $P$ can be defined as

$$
\operatorname{grad} f(P)=\nabla f=\left(\frac{\partial f}{\partial x}, \frac{\partial f}{\partial y}, \frac{\partial f}{\partial z}\right) .
$$

It is known from [26] that combat power potential energy of fighter is a function with regard to coordinates of fighters and targets $\left(x, y, z, x_{T}, y_{T}, z_{T}\right)$. Calculate the gradient of $e$ depicted in [26] on $x_{T}, y_{T}, z_{T}$ and then we can get

$$
\begin{gathered}
\frac{\partial e\left(x_{T}, y_{T}, z_{T}\right)}{\partial x_{T}}=\frac{\partial e_{D}\left(x_{T}, y_{T}, z_{T}\right)}{\partial x_{T}}\left(\omega_{1} e_{W}\left(x_{T}, y_{T}, z_{T}\right)\right. \\
\left.+\omega_{2} e_{E}\left(x_{T}, y_{T}, z_{T}\right)\right)+e_{D}\left(x_{T}, y_{T}, z_{T}\right) \\
\cdot\left(\omega_{1} \frac{\partial e_{W}\left(x_{T}, y_{T}, z_{T}\right)}{\partial x_{T}}+\omega_{2} \frac{\partial e_{E}\left(x_{T}, y_{T}, z_{T}\right)}{\partial x_{T}}\right), \\
\frac{\partial e\left(x_{T}, y_{T}, z_{T}\right)}{\partial y_{T}}=\frac{\partial e_{D}\left(x_{T}, y_{T}, z_{T}\right)}{\partial y_{T}}\left(\omega_{1} e_{W}\left(x_{T}, y_{T}, z_{T}\right)\right. \\
\left.+\omega_{2} e_{E}\left(x_{T}, y_{T}, z_{T}\right)\right)+e_{D}\left(x_{T}, y_{T}, z_{T}\right) \\
\quad\left(\omega_{1} \frac{\partial e_{W}\left(x_{T}, y_{T}, z_{T}\right)}{\partial y_{T}}+\omega_{2} \frac{\partial e_{E}\left(x_{T}, y_{T}, z_{T}\right)}{\partial y_{T}}\right), \\
\frac{\partial e\left(x_{T}, y_{T}, z_{T}\right)}{\partial z_{T}}=\frac{\partial e_{D}\left(x_{T}, y_{T}, z_{T}\right)}{\partial z_{T}}\left(\omega_{1} e_{W}\left(x_{T}, y_{T}, z_{T}\right)\right. \\
\left.+\omega_{2} e_{E}\left(x_{T}, y_{T}, z_{T}\right)\right)+e_{D}\left(x_{T}, y_{T}, z_{T}\right) \\
\quad\left(\omega_{1} \frac{\partial e_{W}\left(x_{T}, y_{T}, z_{T}\right)}{\partial z_{T}}+\omega_{2} \frac{\partial e_{E}\left(x_{T}, y_{T}, z_{T}\right)}{\partial z_{T}}\right) .
\end{gathered}
$$

Take $\partial e\left(x_{T}, y_{T}, z_{T}\right) / \partial x_{T}$, for instance; through further derivation, we will obtain

$$
\begin{aligned}
& \frac{\partial e\left(x_{T}, y_{T}, z_{T}\right)}{\partial x_{T}} \\
& =\frac{\partial\left(\ln \left(1+A_{D R}\left(x_{T}, y_{T}, z_{T}\right)+A_{D I R}\left(x_{T}, y_{T}, z_{T}\right)\right)\right)}{\partial x_{T}} \\
& +\left(\omega_{1} \ln \left(1+A_{M}\left(x_{T}, y_{T}, z_{T}\right)\right)\right. \\
& \left.+\omega_{2} \ln \left(1+A_{D I S}\left(x_{T}, y_{T}, z_{T}\right)\right)\right) \\
& +\ln \left(1+A_{D R}\left(x_{T}, y_{T}, z_{T}\right)+A_{D I R}\left(x_{T}, y_{T}, z_{T}\right)\right) \\
& +\left(\omega_{1} \frac{\partial\left(\ln \left(1+A_{M}\left(x_{T}, y_{T}, z_{T}\right)\right)\right)}{\partial x_{T}}\right. \\
& \left.+\omega_{2} \frac{\partial\left(\ln \left(1+A_{D I S}\left(x_{T}, y_{T}, z_{T}\right)\right)\right)}{\partial x_{T}}\right) .
\end{aligned}
$$

For losing the burden of calculation, $A_{D R}\left(x_{T}, y_{T}, z_{T}\right)$, $A_{D I R}\left(x_{T}, y_{T}, z_{T}\right), A_{M}\left(x_{T}, y_{T}, z_{T}\right), A_{D I S}\left(x_{T}, y_{T}, z_{T}\right)$ are simplified as $A_{D R}\left(x_{T}, y_{T}, z_{T}\right)=C_{1} / r\left(x_{T}, y_{T}, z_{T}\right), A_{D I R}\left(x_{T}, y_{T}\right.$, $\left.z_{T}\right)=C_{2} / r\left(x_{T}, y_{T}, z_{T}\right), A_{M}\left(x_{T}, y_{T}, z_{T}\right)=C_{3} / r\left(x_{T}, y_{T}, z_{T}\right)$, $A_{D I S}\left(x_{T}, y_{T}, z_{T}\right)=C_{4} / r\left(x_{T}, y_{T}, z_{T}\right)$, where $r\left(x_{T}, y_{T}, z_{T}\right)=$ $\sqrt{\left(x_{A}-x_{T}\right)^{2}+\left(y_{A}-y_{T}\right)^{2}+\left(z_{A}-z_{T}\right)^{2}}$. Substitute simplified $A_{D R}\left(x_{T}, y_{T}, z_{T}\right), A_{D I R}\left(x_{T}, y_{T}, z_{T}\right), A_{M}\left(x_{T}, y_{T}, z_{T}\right), A_{D I S}\left(x_{T}\right.$, $y_{T}, z_{T}$ ) into (3); we will derive

$$
\begin{aligned}
& \frac{\partial e\left(x_{T}, y_{T}, z_{T}\right)}{\partial x_{T}} \\
& =\frac{\partial\left(\ln \left(1+C_{1} / r\left(x_{T}, y_{T}, z_{T}\right)+C_{2} / r\left(x_{T}, y_{T}, z_{T}\right)\right)\right)}{\partial x_{T}} \\
& \cdot\left(\omega_{1} \ln \left(1+\frac{C_{3}}{r\left(x_{T}, y_{T}, z_{T}\right)}\right)+\omega_{2}\right. \\
& \left.\quad \cdot \ln \left(1+\frac{C_{4}}{r\left(x_{T}, y_{T}, z_{T}\right)}\right)\right)+\ln \left(1+\frac{C_{1}}{r\left(x_{T}, y_{T}, z_{T}\right)}\right. \\
& \left.+\frac{C_{2}}{r\left(x_{T}, y_{T}, z_{T}\right)}\right) \\
& .\left(\omega_{1} \frac{\partial\left(\ln \left(1+C_{3} / r\left(x_{T}, y_{T}, z_{T}\right)\right)\right)}{\partial x_{T}}\right. \\
& \left.+\omega_{2} \frac{\partial\left(\ln \left(1+C_{4} / r\left(x_{T}, y_{T}, z_{T}\right)\right)\right)}{\partial x_{T}}\right)
\end{aligned}
$$




$$
\begin{aligned}
& =\frac{\partial\left(\ln \left(1+C_{1} / r\left(x_{T}, y_{T}, z_{T}\right)+C_{2} / r\left(x_{T}, y_{T}, z_{T}\right)\right)\right)}{\partial r\left(x_{T}, y_{T}, z_{T}\right)} \\
& \frac{\left(x_{A}-x_{T}\right) \cdot C_{3}}{\left(r\left(x_{T}, y_{T}, z_{T}\right)+C_{3}\right) \cdot r^{2}\left(x_{T}, y_{T}, z_{T}\right)}+\omega_{2} \\
& \frac{\partial r\left(x_{T}, y_{T}, z_{T}\right)}{\partial x_{T}} \cdot\left(\omega_{1} \ln \left(1+\frac{C_{3}}{r\left(x_{T}, y_{T}, z_{T}\right)}\right)\right. \\
& \left.\cdot\left(\frac{\left(x_{A}-x_{T}\right) \cdot C_{4}}{\left(r\left(x_{T}, y_{T}, z_{T}\right)+C_{4}\right) \cdot r^{2}\left(x_{T}, y_{T}, z_{T}\right)}\right)\right) . \\
& \left.+\omega_{2} \ln \left(1+\frac{C_{4}}{r\left(x_{T}, y_{T}, z_{T}\right)}\right)\right)+\ln (1 \\
& \left.+\frac{C_{1}}{r\left(x_{T}, y_{T}, z_{T}\right)}+\frac{C_{2}}{r\left(x_{T}, y_{T}, z_{T}\right)}\right) \cdot\left(\omega_{1}\right. \\
& \cdot \frac{\partial\left(\ln \left(1+C_{3} / r\left(x_{T}, y_{T}, z_{T}\right)\right)\right)}{\partial r\left(x_{T}, y_{T}, z_{T}\right)} \cdot \frac{\partial r\left(x_{T}, y_{T}, z_{T}\right)}{\partial x_{T}} \\
& +\omega_{2} \cdot \frac{\partial\left(\ln \left(1+C_{4} / r\left(x_{T}, y_{T}, z_{T}\right)\right)\right)}{\partial r\left(x_{T}, y_{T}, z_{T}\right)} \\
& \left.\cdot \frac{\partial r\left(x_{T}, y_{T}, z_{T}\right)}{\partial x_{T}}\right)=\left(\frac{r\left(x_{T}, y_{T}, z_{T}\right)}{r\left(x_{T}, y_{T}, z_{T}\right)+C_{1}+C_{2}}\right. \\
& \left.\cdot-\frac{C_{1}+C_{2}}{r^{2}\left(x_{T}, y_{T}, z_{T}\right)} \cdot \frac{2\left(x_{A}-x_{T}\right)}{2 r\left(x_{T}, y_{T}, z_{T}\right)} \cdot-1\right) \cdot\left(\omega_{1}\right. \\
& \cdot \ln \left(1+\frac{C_{3}}{r\left(x_{T}, y_{T}, z_{T}\right)}\right)+\omega_{2} \\
& \left.\cdot \ln \left(1+\frac{C_{4}}{r\left(x_{T}, y_{T}, z_{T}\right)}\right)\right)+\ln \left(1+\frac{C_{1}}{r\left(x_{T}, y_{T}, z_{T}\right)}\right. \\
& \left.+\frac{C_{2}}{r\left(x_{T}, y_{T}, z_{T}\right)}\right) \cdot\left(\omega _ { 1 } \cdot \left(\frac{r\left(x_{T}, y_{T}, z_{T}\right)}{r\left(x_{T}, y_{T}, z_{T}\right)+C_{3}}\right.\right. \\
& \left.\cdot-\frac{C_{3}}{r^{2}\left(x_{T}, y_{T}, z_{T}\right)} \cdot \frac{2\left(x_{A}-x_{T}\right)}{2 r\left(x_{T}, y_{T}, z_{T}\right)} \cdot-1\right)+\omega_{2} \\
& \cdot\left(\frac{r\left(x_{T}, y_{T}, z_{T}\right)}{r\left(x_{T}, y_{T}, z_{T}\right)+C_{4}} \cdot-\frac{C_{4}}{r^{2}\left(x_{T}, y_{T}, z_{T}\right)}\right. \\
& \left.\left.\cdot \frac{2\left(x_{A}-x_{T}\right)}{2 r\left(x_{T}, y_{T}, z_{T}\right)} \cdot-1\right)\right) \\
& =\frac{\left(x_{A}-x_{T}\right) \cdot\left(C_{1}+C_{2}\right)}{\left(r\left(x_{T}, y_{T}, z_{T}\right)+C_{1}+C_{2}\right) \cdot r^{2}\left(x_{T}, y_{T}, z_{T}\right)} \cdot\left(\omega_{1}\right. \\
& \cdot \ln \left(1+\frac{C_{3}}{r\left(x_{T}, y_{T}, z_{T}\right)}\right)+\omega_{2} \\
& \left.\cdot \ln \left(1+\frac{C_{4}}{r\left(x_{T}, y_{T}, z_{T}\right)}\right)\right)+\ln (1 \\
& \left.+\frac{C_{1}+C_{2}}{r\left(x_{T}, y_{T}, z_{T}\right)}\right) \cdot\left(\omega_{1}\right. \\
& \begin{array}{l}
\frac{\partial e\left(x_{T}, y_{T}, z_{T}\right)}{\partial y_{T}} \\
=\frac{\left(y_{A}-y_{T}\right) \cdot\left(C_{1}+C_{2}\right)}{\left(r\left(x_{T}, y_{T}, z_{T}\right)+C_{1}+C_{2}\right) \cdot r^{2}\left(x_{T}, y_{T}, z_{T}\right)} \cdot\left(\omega_{1}\right. \\
\cdot \ln \left(1+\frac{C_{3}}{r\left(x_{T}, y_{T}, z_{T}\right)}\right)+\omega_{2} \\
\left.\cdot \ln \left(1+\frac{C_{4}}{r\left(x_{T}, y_{T}, z_{T}\right)}\right)\right)+\ln (1 \\
\left.+\frac{C_{1}+C_{2}}{r\left(x_{T}, y_{T}, z_{T}\right)}\right) \cdot\left(\omega_{1}\right. \\
\cdot \frac{\left(y_{A}-y_{T}\right) \cdot C_{3}}{\left(r\left(x_{T}, y_{T}, z_{T}\right)+C_{3}\right) \cdot r^{2}\left(x_{T}, y_{T}, z_{T}\right)}+\omega_{2} \\
\left.\quad\left(\frac{\left(y_{A}-y_{T}\right) \cdot C_{4}}{\left(r\left(x_{T}, y_{T}, z_{T}\right)+C_{4}\right) \cdot r^{2}\left(x_{T}, y_{T}, z_{T}\right)}\right)\right)
\end{array} \\
& \frac{\partial e\left(x_{T}, y_{T}, z_{T}\right)}{\partial z_{T}} \\
& =\frac{\left(z_{A}-z_{T}\right) \cdot\left(C_{1}+C_{2}\right)}{\left(r\left(x_{T}, y_{T}, z_{T}\right)+C_{1}+C_{2}\right) \cdot r^{2}\left(x_{T}, y_{T}, z_{T}\right)} \cdot\left(\omega_{1}\right. \\
& \cdot \ln \left(1+\frac{C_{3}}{r\left(x_{T}, y_{T}, z_{T}\right)}\right) \\
& \left.+\omega_{2} \ln \left(1+\frac{C_{4}}{r\left(x_{T}, y_{T}, z_{T}\right)}\right)\right)+\ln (1 \\
& \left.+\frac{C_{1}+C_{2}}{r\left(x_{T}, y_{T}, z_{T}\right)}\right) \cdot\left(\omega_{1}\right. \\
& \cdot \frac{\left(z_{A}-z_{T}\right) \cdot C_{3}}{\left(r\left(x_{T}, y_{T}, z_{T}\right)+C_{3}\right) \cdot r^{2}\left(x_{T}, y_{T}, z_{T}\right)}+\omega_{2} \\
& \left.\cdot\left(\frac{\left(z_{A}-z_{T}\right) \cdot C_{4}}{\left(r\left(x_{T}, y_{T}, z_{T}\right)+C_{4}\right) \cdot r^{2}\left(x_{T}, y_{T}, z_{T}\right)}\right)\right) \text {. }
\end{aligned}
$$


Descending velocity of fighter $A$ in direction of target $T$ is got in the light of definition of gradient:

$$
v_{d\left(x_{A}, y_{A}, z_{A}\right)}\left(x_{T}, y_{T}, z_{T}\right)=\left|\operatorname{grad}\left(e\left(x_{T}, y_{T}, z_{T}\right)\right)\right|=\sqrt{\left(\frac{\partial e\left(x_{T}, y_{T}, z_{T}\right)}{\partial x_{T}}\right)^{2}+\left(\frac{\partial e\left(x_{T}, y_{T}, z_{T}\right)}{\partial y_{T}}\right)^{2}+\left(\frac{\partial e\left(x_{T}, y_{T}, z_{T}\right)}{\partial z_{T}}\right)^{2}}
$$

Similarly, descending velocity of target $T$ in direction of fighter $A$ is given as

$$
v_{d\left(x_{T}, y_{T}, z_{T}\right)}\left(x_{A}, y_{A}, z_{A}\right)=\left|\operatorname{grad}\left(e\left(x_{A}, y_{A}, z_{A}\right)\right)\right|=\sqrt{\left(\frac{\partial e\left(x_{A}, y_{A}, z_{A}\right)}{\partial x_{A}}\right)^{2}+\left(\frac{\partial e\left(x_{A}, y_{A}, z_{A}\right)}{\partial y_{A}}\right)^{2}+\left(\frac{\partial e\left(x_{A}, y_{A}, z_{A}\right)}{\partial z_{A}}\right)^{2}} .
$$

2.1.3. Calculation on Relative Superiority. Calculation on relative superiority of fighter $i$ with respect to target $j$ is expressed as

$$
\begin{aligned}
& p_{i j} \\
& =\frac{v_{d\left(x_{A_{i}}, y_{A_{i}}, z_{A_{i}}\right)}\left(x_{T_{j}}, y_{T_{j}}, z_{T_{j}}\right)}{v_{d\left(x_{A_{i}}, y_{A_{i}}, z_{A_{i}}\right)}\left(x_{T_{j}}, y_{T_{j}}, z_{T_{j}}\right)+v_{d\left(x_{T_{j}}, y_{T_{j}}, z_{T_{j}}\right)}\left(x_{A_{i}}, y_{A_{i}}, z_{A_{i}}\right)},
\end{aligned}
$$

where $v_{d\left(x_{A_{i}}, y_{A_{i}}, z_{A_{i}}\right)}\left(x_{T_{j}}, y_{T_{j}}, z_{T_{j}}\right)$ is the descending velocity of fighter $i$ in direction to location of target $j$ and $v_{d\left(x_{T_{j}}, y_{T_{j}}, z_{T_{j}}\right)}\left(x_{A_{i}}, y_{A_{i}}, z_{A_{i}}\right)$ is the descending velocity of target $j$ in direction to location of fighter $i$.

2.2. WTA Formulation. In the paper, the problem is formulated as a combinatorial optimization problem, with the objective of minimizing the total lethality of the targets. The fundamental problem variable is relative superiority $p_{i j}$.

Consider the assignment problem of $m$ fighters to $n$ targets: let $p_{i j} \in[0,1]$ be the relative superiority, where $i \in$ $\{1,2, \ldots, m\}$ and $j \in\{1,2, \ldots, n\}$. The WTA is formulated as follows:

$$
\begin{aligned}
J=\min & \sum_{j=1}^{n}\left(\prod_{i=1}^{m}\left(1-p_{i j}\right)^{x_{i j}}\right) \\
\text { s.t. } & \sum_{i=1}^{m} x_{i j} \geq 1, \\
& \sum_{j=1}^{n} x_{i j} \leq 1,
\end{aligned}
$$

where $x_{i j}$ is the decision value indicating if fighter $i$ is assigned to target $j ; x_{i j}=1$ if fighter $i$ is assigned to target $j$ and $x_{i j}=0$ if fighter $i$ is not assigned to target $j$.

In the above formulation, we minimize the sum of the weighted cumulative probabilities of threat lethality while ensuring that all constraints are satisfied. Two constraints, respectively, denote that each target must be assigned by one fighter and each fighter can be assigned to one target at most.

\section{A Hybrid Algorithm for the Problem}

3.1. Improvements on AFSA. To improve the performance of the algorithm, several modifications were introduced into the AFSA, including initialization, visual and movement strategy, and leap behavior. Concrete implementations are presented below.

3.1.1. Initialization. Initial solution causes crucial influences on convergence performance of the algorithm. The initial solution is randomly generated in the most existed study, by which it is difficult to get solution of high quality. Therefore a hybrid initialization method involving chaos, information entropy, and opposition-based learning methods is proposed and described in Figure 1.

Assume that there are $p$ artificial fish in the swarm, each of which has $q$ variables. $X_{r s}$ denotes the variable $s$ within fish $r, r \in\{1, \ldots, p\}, s \in\{1, \ldots, q\}$. The pseudocode for two algorithms in Figure 1 is described in Algorithms 1 and 2.

3.1.2. Pseudo-Parallel Evolution. In the late period of search, artificial fish will move more slowly and be misled to local optimums. A pseudo-parallel evolution strategy dividing the artificial fish swarm into multiple subswarms is introduced for solving the problem. Each subswarm executes search for optimum independently and exchanges the optimal solution with others at every iteration to maintain the search space and prevent prematurity.

3.1.3. Artificial Fish Moving Strategy. To improve the search ability of the artificial fish, a moving strategy combining 


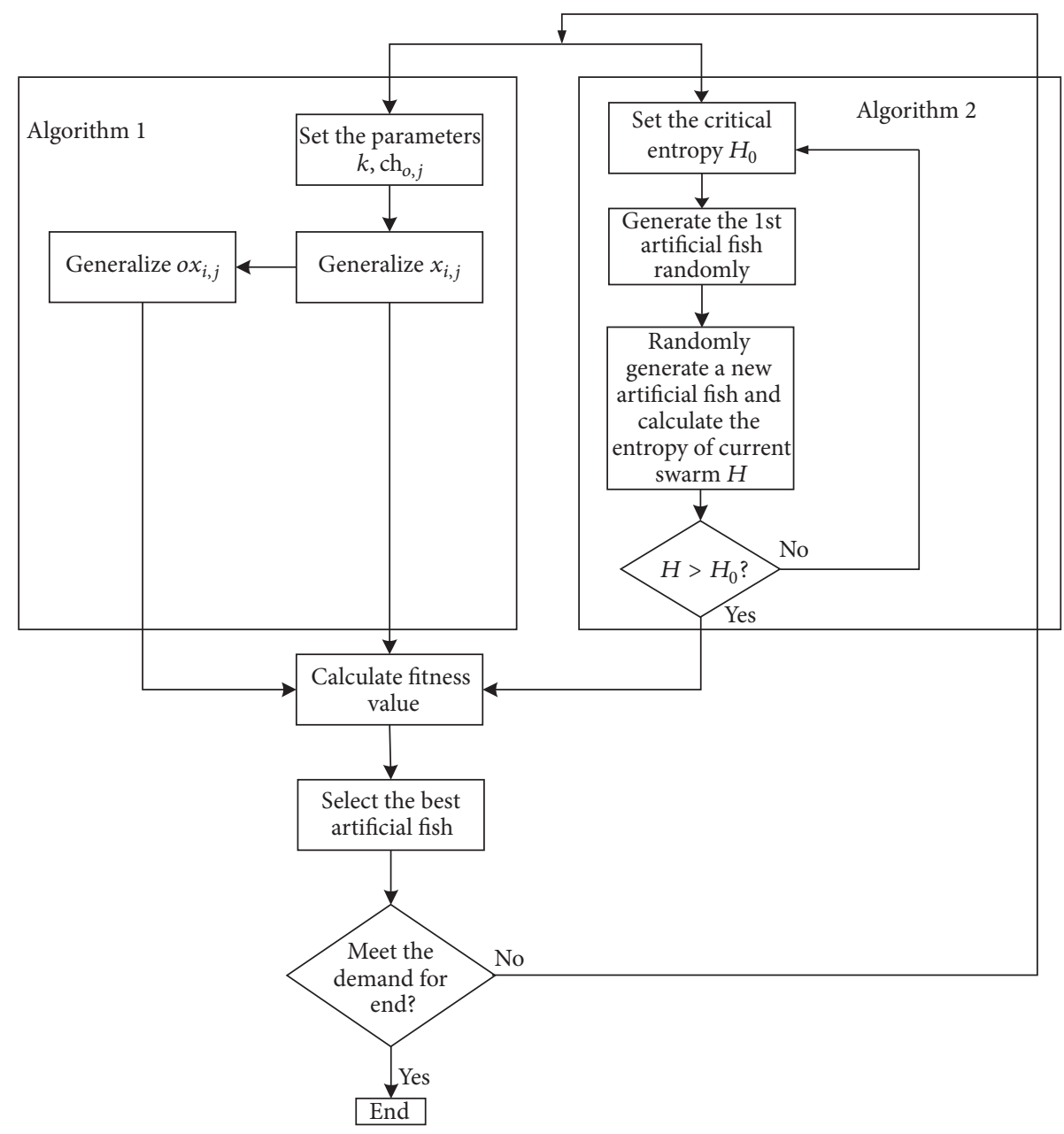

FIgURE 1: Flow of initialization.

maintaining the self-advantage with absorbing the advantage from others is proposed below:

$$
\begin{aligned}
X_{r}^{\text {gen+1 }}= & \frac{\text { gen }}{\text { max gen }} X_{r}^{\text {gen }} \\
& +\frac{\text { max gen }- \text { gen }}{\text { max gen }}\left(X_{u}^{\text {gen }}-X_{r}^{\text {gen }}\right),
\end{aligned}
$$

where gen is the current iteration, max gen is the maximum iteration, $X_{r}^{\text {gen+1 }}, X_{r}^{\text {gen }}$ are the position of artificial fish $r$ at iteration gen +1 and gen, respectively, and $X_{u}^{\text {gen }}$ is the position of artificial fish $u$ at gen iteration. The first part, (gen/max gen) $X_{r}^{\text {gen }}$, describes artificial fish keeping the selfsuperiority; the second part inspired by neighborhood search strategy, ((max gen - gen $) / \max$ gen $)\left(X_{u}^{\text {gen }}-X_{r}^{\text {gen }}\right)$, indicates the absorbance of advantage from another artificial fish $u$ within visual range.

3.1.4. Self-Adaptive Visual Procedure. Visual, which has been deeply studied, determines the convergence speed and accuracy of AFSA. The modifications on visual have been proposed by many researches, as given in (11) (13). Reference [19] proposed an improved visual formulation:

$$
\begin{aligned}
\text { visual }^{\text {gen }}= & \text { visual }^{\text {gen-1 }} \\
& \cdot\left(L_{\text {low }}+\left(\text { rand } \cdot\left(L_{\text {high }}-L_{\text {low }}\right)\right)\right),
\end{aligned}
$$

where visual $^{\text {gen }}$ and visual ${ }^{\text {gen-1 }}$ stand for the visual at current iteration gen and previous iteration gen $-1 . L_{\text {high }}$ and $L_{\text {low }}$ represent the upper and lower bounds of the visual, respectively.

The model utilizes a random number to control the visual change and to ensure the overall trend getting lower, whereas, due to stochastic factors existing during the evolution, it is incapable of precise adjustment to the visual.

Another modification on visual is presented in the following equation [20]:

$$
\text { visual }^{\text {gen }}=\text { visual }^{\text {gen-1 }} \cdot \frac{\left|y_{\text {best }}-\bar{y}\right|}{y_{\text {best }}},
$$

where $y_{\text {best }}$ is the global best solution by estimation and $\bar{y}$ is the mean solution. 

(1) Initialize parameter: Set the maximum chaotic iteration number ingen
(2) for $r=1: p$ do
(3) for $s=1: q$ do
(4) for $o=1$ : ingen do
(5) $\mathrm{ch}_{o s}=\sin \left(\pi \cdot \mathrm{ch}_{o-1, s}\right) \quad \%$ Update the chaotic factor
(6) end for
(7) $X_{o s}=X_{\min , s}+\operatorname{ch}_{o s}\left(X_{\max , s}-X_{\min , s}\right) \quad \%$ Generate initial chaotic solution
(8) $O X_{o s}=X_{\min , s}+X_{\max , s}-X_{r s} \%$ Generate initial opposition-based learning solution
(9) end for
(10) end for

Algorithm 1: Initialization based on logistic chaos and oppositionbased learning [27, 28].
(1) Set the critical entropy $H_{0}$
(2) $H=0$
(3) while $H<H_{0}$
(4) for $r=1: p$
(5) for $t=r+1: p$
(6) for $s=1: q$
(7) $X_{t s}=X_{\min , s}+$ rand $\cdot\left(X_{\max , s}-X_{\min , s}\right)$
(8) $P_{r t}^{s}=1-\left|X_{r s}-X_{t s}\right| /\left(A_{s}^{\max }-A_{s}^{\mathrm{min}}\right) \quad \%$ Difference ratio Between $X_{r s}$ and $X_{t s}, A_{s}^{\max }$ and $A_{s}^{\min }$ are the maximum and minimum value of the variable $s$ respectively
(9) $H_{s}=\sum_{r=1}^{p} \sum_{t=2}^{p}\left(-P_{r t}^{s} \log P_{r t}^{s}\right) \quad \%$ Entropy of variable $s$ in artificial fish swarm
(10) $H=(1 / q) \sum_{s=1}^{q} H_{s} \quad \%$ Entropy of the whole swarm
(11) end for
(12) end for
(13) end for
(14) end while

Algorithm 2: Initialization based on information entropy [29].
In accordance with (12), the mean solution is getting closer to the global best solution by estimation. During the approach to the optimal solution, difference between the current mean solution and the optimal one decreases, as well as the visual scope. However, $y_{\text {best }}$ is hard to estimate, which easily causes the algorithm to fall into local optimal or poor performance in convergence.

Reference [21] proposed another kind of improvement on visual:

$$
\begin{aligned}
& \text { visual }^{\text {gen }} \\
& = \begin{cases}\left(\beta-\frac{\text { gen }}{N}\right) \cdot \text { visual }^{\text {fixed }} \\
\alpha \cdot \text { visual }^{\text {gen }-1}, & \alpha=\left(\frac{\text { visual }^{N}}{\text { visual }^{1}}\right)^{1 / N},\end{cases}
\end{aligned}
$$

where $N$ is the maximum iteration, visual ${ }^{1}$ is the initial value, visual ${ }^{N}$ is the previously set value at the last iteration, visual $^{\text {fixed }}$ is a fixed value, and $\beta$ is a constant number.

In initial iterations, the visual varies in accordance with the upper formulation for ensuring good performance in convergence speed and global searching, thereafter changing by means of the next one for better convergence accuracy. But there are two deficiencies that existed; the switch condition from the upper formulation to the one below is not quite clear and the visual at the last iteration is not easy to obtain.

The main purpose is to ensure the artificial fish searching with larger visual within initial iterations and with smaller visual afterwards, although the modified means are quite different such as (11) to (13). Whereas the above improvements on visual are insufficient for the research on the algorithm falling into the local optimum, therefore a modified visual model concerned with iteration and convergence speed is constructed as follows:

$$
\text { visual }_{r}^{\text {gen }}= \begin{cases}\max \left\{\text { visual } \cdot \mid D_{r}(\text { gen })-D_{r}(\text { gen }-1) \mid \cdot e^{-30(\text { gen } / \text { max gen })^{s}}, 1\right\}, & D_{r}(\text { gen })-D_{r}(\text { gen }-1) \neq 0, \\ \text { visual } \cdot \mid D_{r}\left(\text { gen }- \text { gen }^{\prime}\right)-D_{r}\left(\text { gen }- \text { gen }^{\prime}-1\right) \mid \cdot e^{-30(\text { gen } / \max \text { gen })^{s}}, & D_{r}(\text { gen })-D_{r}(\text { gen }-1)=0,\end{cases}
$$

where gen' measures the iterations at which the optimal solution stays unchanged, visual is the initial visual, $D_{r}\left(\right.$ gen $\left._{\text {last }}\right)$ is the solution at the last iteration before $D_{r}($ gen $)-D_{r}($ gen -1$)=$ $0, D_{r}\left(\operatorname{gen}_{\text {last }}-1\right)$ is the solution at the previous iteration before gen last $_{\text {as }}$, and $S$ is a constant number that could be $1,3,5$, 10.

From (14) we can see that adjustment in visual changes one dimension at least and gets smaller as $D_{r}$ (gen) $-D_{r}$ (gen 1) decreases, which helps improve the accuracy of the solutions. When $D_{r}$ (gen) $-D_{r}($ gen -1$)=0$, the visual obtained by (14) will get larger, by which artificial fish are capable of escaping from local optimal regions.
3.1.5. Improvements on Leap Behavior. For preventing the artificial fish from converging to local optimal solutions, leap behavior forcing the artificial fish to change its location is adopted in certain references [22, 23]. In this paper, artificial fish executes leap behavior when the optimal solution stands still after one iteration. Meanwhile, it is thought that the higher the number of iterations at which optimal solution stagnates is, the higher the extent the optimal solution varies and the higher the probability of leap behavior execution is. Accordingly, improvements on leap behavior probability and leap behavior strategy are designed as follows. 
The leap behavior is modified as outlined in (15):

$$
X_{r}^{\text {gen }+1}=X_{r}^{\text {gen }}+\operatorname{visual}_{r}^{\text {gen }} \cdot \lambda \cdot \text { rand, }
$$

where $\lambda$ is a bool vector; the quantity of 1 in vector is obtained from

$$
\begin{aligned}
& \theta=\max \{1, q-q \\
& \left.\cdot \operatorname{round}\left(e^{\left.-30 \text { gen }^{\prime} / \text { gen }\right)^{s}} \cdot \frac{D_{r}\left(\text { gen }- \text { gen }^{\prime}\right)}{D_{r}(\text { gen })}\right)\right\} .
\end{aligned}
$$

Equation (16) makes it certain that one dimension at least, $q$ dimension at most in location of artificial fish $r$, changes by leap behavior at each iteration. The bigger $D_{r}\left(\right.$ gen - gen $\left.{ }^{\prime}\right) / D_{r}$ (gen) and gen'/gen are, the more dimension changes. By this way, leap behavior is able to adjust the location of artificial fish dynamically and to achieve the goal of avoiding premature convergence.

3.2. Improvement on HS. In this article, improvements on HS contains two domains: harmony memory consideration rate and new harmony generating strategy.

\subsubsection{Improvements on Harmony Memory Consideration}

Rate. Modifications on harmony memory consideration rate have been studied in many researches [30, 31]. Through the reference, it can be concluded that harmony memory consideration rate should be given a small value to increase the diversity of the harmony memory and global search when solutions differ greatly. On the other hand, when the solutions are relatively resembled, harmony memory consideration rate should be raised for performing search more accurately and reducing the time for searching. In this paper, it is considered that iteration has effect on harmony memory consideration rate as solution does; particularly harmony memory consideration rate is supposed to increase gradually at final iterations for acting better in global search. The harmony memory consideration rate is formulated from the perspectives of current iteration and deviations between harmony vectors as follows:

$$
\operatorname{HMCR}(\text { gen })=\frac{E_{\min }(\text { gen })}{E_{\max }(\text { gen })} e^{\text {gen } / \text { max gen }-1},
$$

where $E_{\min }$ (gen), $E_{\max }$ (gen), respectively, represent the best and worst solution in harmony memory at iteration gen. The harmony memory consideration rate in (17) utilizes dynamic mechanism to select the harmony vector under various circumstances. The larger $E_{\min }$ (gen) $/ E_{\max }$ (gen) is, the smaller the difference among the harmony vectors in harmony memory is, which indicates that HMCR(gen) becomes larger to converge to the optimum fastly. Likewise, the larger gen is, the larger HMCR(gen) is, and the algorithm is able to have a faster convergence to the optimum.

3.2.2. New Harmony Improvisation. New harmony improvisation deciding general performance in optimization is also seen as the key point in modifications on HS. A new method for harmony improvisation in terms of learning strategy is defined in this paper, which is described as follows:

$$
u_{s}^{\text {gen }}= \begin{cases}u_{r_{1}, s}^{\text {gen }}+\min \left\{c_{1}, c_{2}\right\} \cdot\left(u_{\text {best }, s}^{\text {gen }}-u_{r_{1}, s}^{\text {gen }}\right)+\max \left\{c_{1}, c_{2}\right\} \cdot\left(u_{r_{2}, s}^{\text {gen }}-u_{r_{1}, s}^{\text {gen }}\right), & D_{\text {best }}(\text { gen })-D_{\text {best }}(\text { gen }-1)=0 \\ u_{r_{1}, s}^{\text {gen }}+\max \left\{c_{1}, c_{2}\right\} \cdot\left(u_{\text {best }, s}^{\text {gen }}-u_{r_{1}, s}^{\text {gen }}\right)+\min \left\{c_{1}, c_{2}\right\} \cdot\left(u_{r_{2}, s}^{\text {gen }}-u_{r_{1}, s}^{\text {gen }}\right), & D_{\text {best }}(\text { gen })-D_{\text {best }}(\text { gen }-1) \neq 0\end{cases}
$$

where $u_{s}^{\text {gen }}$ is the decision variable $s$ in harmony vector generated at iteration gen, $u_{r_{1}, s}^{\text {gen }}, u_{r_{2}, s}^{\text {gen }}, u_{\text {best,s }}^{\text {gen }}$ are the decision variables $s$ in harmony vectors $r_{1}, r_{2}$ and the optimal harmony vector in harmony memory generated at iteration gen, and $c_{1}$, $c_{2}$ are learning coefficients, $0 \leq c_{1}, c_{2} \leq 1, c_{1} \neq c_{2}$.

The bigger one between $c_{1}$ and $c_{2}$ is selected to make sure that the harmony is affected by the neighborhood solution inside the harmony memory and avoid prematurity when $D_{\text {best }}($ gen $)-D_{\text {best }}($ gen -1$)=0$. Otherwise, the harmony improvisation is more influenced by the optimal solution, which helps in converging to optimum more quickly.

\subsection{Switch Condition}

3.3.1. Switch Condition Analysis. Switch condition acting as a key part of the hybrid algorithm causes a great influence on speed and accuracy of convergence. Switch condition is discussed by two aspects as switching iteration and individual standard error of artificial fish.
(1) Switching Iteration. Switching iteration is to make the hybrid algorithm switch from improved AFSA to improved $\mathrm{HS}$ at a certain iteration, which is obtained by multiple concrete experiments.

(2) Individual Standard Error of Artificial Fish. This factor reflects the deviation among artificial fish in the swarm. It should be switched to the HS for the following search while the individual standard error of artificial fish gets smaller, which means the search space gets smaller. The model of individual standard error of artificial fish, with regard to location and fitness value, is proposed as follows.

The standard error of location on artificial fish $l$ se is calculated by

$$
l s e=\sqrt{\frac{1}{q-1} \sum_{s=1}^{q} l \mathrm{se}_{s}^{2}}
$$




$$
l \mathrm{se}_{s}^{2}=\frac{1}{p-1} \sum_{r=1}^{p}\left(X_{r s}-\bar{X}_{s}\right)^{2}
$$

where $\bar{X}_{s}$ is the average location of all artificial fish in dimension $s$. The artificial fish are of high probability to gather in local optimal region while each fish gets closer, as well as $l$ se being smaller.

The standard error of fitness value on artificial fish $f$ se is calculated by

$$
f \text { se }=\sqrt{\sum_{r=1}^{p} \frac{\left(D_{r}(\text { gen })-\bar{D}(\text { gen })\right)^{2}}{p-1}},
$$

where $\bar{D}$ (gen) is the average fitness value. Likewise, the artificial fish seems to get trapped in the local optimum area while fitness value of each fish is resembled, as well as $f$ se being smaller.

From the information mentioned above, the individual standard error of artificial fish is derived by

$$
\begin{aligned}
\text { se }= & \varphi_{1} \cdot l \text { se }+\varphi_{2} \cdot f \text { se } \\
\text { s.t. } & 0 \leq \varphi_{1}, \varphi_{2} \leq 1 \\
& \varphi_{1}+\varphi_{2}=1,
\end{aligned}
$$

where $\varphi_{1}, \varphi_{2}$, respectively, represent the coefficients about $l$ se and $f$ se. The switch to improved HS should be executed for global optimum if se is smaller than the presented value se ${ }_{\text {def }}$, which means the stand error of fitness value and location fluctuate to a smaller extent; therefore the solution is weak.

3.3.2. Design for Switch Step. For the sake of fast convergence and quality of solutions, it is ruled in this paper that the switch is executed only if any condition is satisfied. Due to the different performances of the algorithms when facing various problems or benchmark functions, it is also regulated that the individual standard error of artificial fish is seen as the prior factor, and the switch flow is given in Figure 2.

It can be seen from Figure 2 that individual standard error of artificial fish is investigated at first; switch is executed if satisfied; otherwise checking whether the algorithm is qualified to switch from the perspective of switching iteration, the switch is carried on if satisfied, or else the algorithm runs back to the improved artificial fish swarm algorithm.

3.4. Flow for Hybrid Algorithm. The concrete flow for the proposed hybrid algorithm is depicted in Figure 3.

\section{Simulation Results}

4.1. Verification Tests by Benchmark Functions. The algorithms are always tested by benchmark functions. For thoroughly validating the performances of the proposed IAFSAHIS, other three algorithms including IABHS, IBAFSA, and GDHS are introduced for comparison. Main properties of the

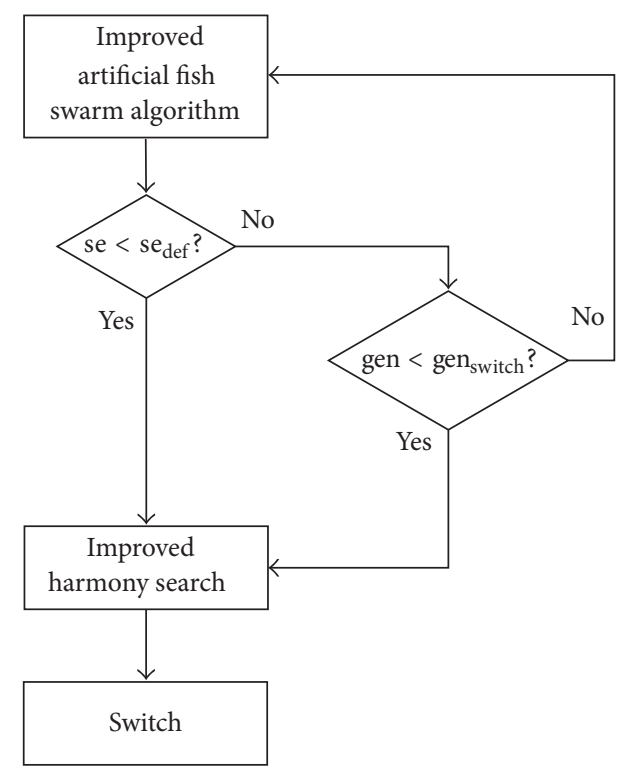

FIGURE 2: Switch flow for algorithm.

selected benchmark functions used in the experiments are shown in Table 1.

Parameters are set as follows: ingen $=100, H_{0}=0.3$, $S=5$, visual $=1.5, \gamma_{1}=0.3, \gamma_{2}=0.5, c_{1}=0.6, c_{2}=0.4$, $\varphi_{1}=0.6, \varphi_{2}=0.4$, $\max$ gen $=100000$, and $n=30$; for other parameters, refer to $[24,30,32]$.

The algorithms were run 100 times independently to catch their stochastic properties and the results are depicted in Table 2 and Figures 4-11.

The parameters Best, Mean, Worst, and Std.dev are the best, mean, worst, and stand error of the optimum on average after 100 running times, where Mean reflects the precision of the results obtained by the algorithms within certain iterations and Std.dev reflects the robustness and stability of the algorithms.

As seen from Table 2 and Figures 4-11, the advantages in terms of best, median, worst, mean, and standard error of IAFSA-HIS are outstanding among the four algorithms in general. Particularly, IAFSA-IHS achieves the theoretical optimum while others fail still the termination in $f_{6}$ and $f_{7}$. However, the proposed algorithm performs relatively poor with regard to $f_{1}, f_{4}, f_{5}$, especially for $f_{1}$. Compared with GDHS obtaining the theoretical optimum within fewer iterations in $f_{1}$, IAFSA-IHS fails to get the satisfying solution, which seems to fall into the prematurity as IABHS and IBAFSA do.

It can be concluded that IAFSA-IHS exactly performs better on robustness, convergence speed, and precision with the improvements mentioned above synthetically. Taking $f_{6}$, for instance, as a multimodal function, there exist a lot of local optima surrounding the global optimum which easily misguides the algorithm to stay in the local optimum. For solving the problem, GDHS sets HMCR, PAR, $b w$ in dynamical mode, IBAFSA decodes the artificial fish into binary, sets the trial point, and reinitializes the artificial fish 
TABLE 1: Benchmark functions.

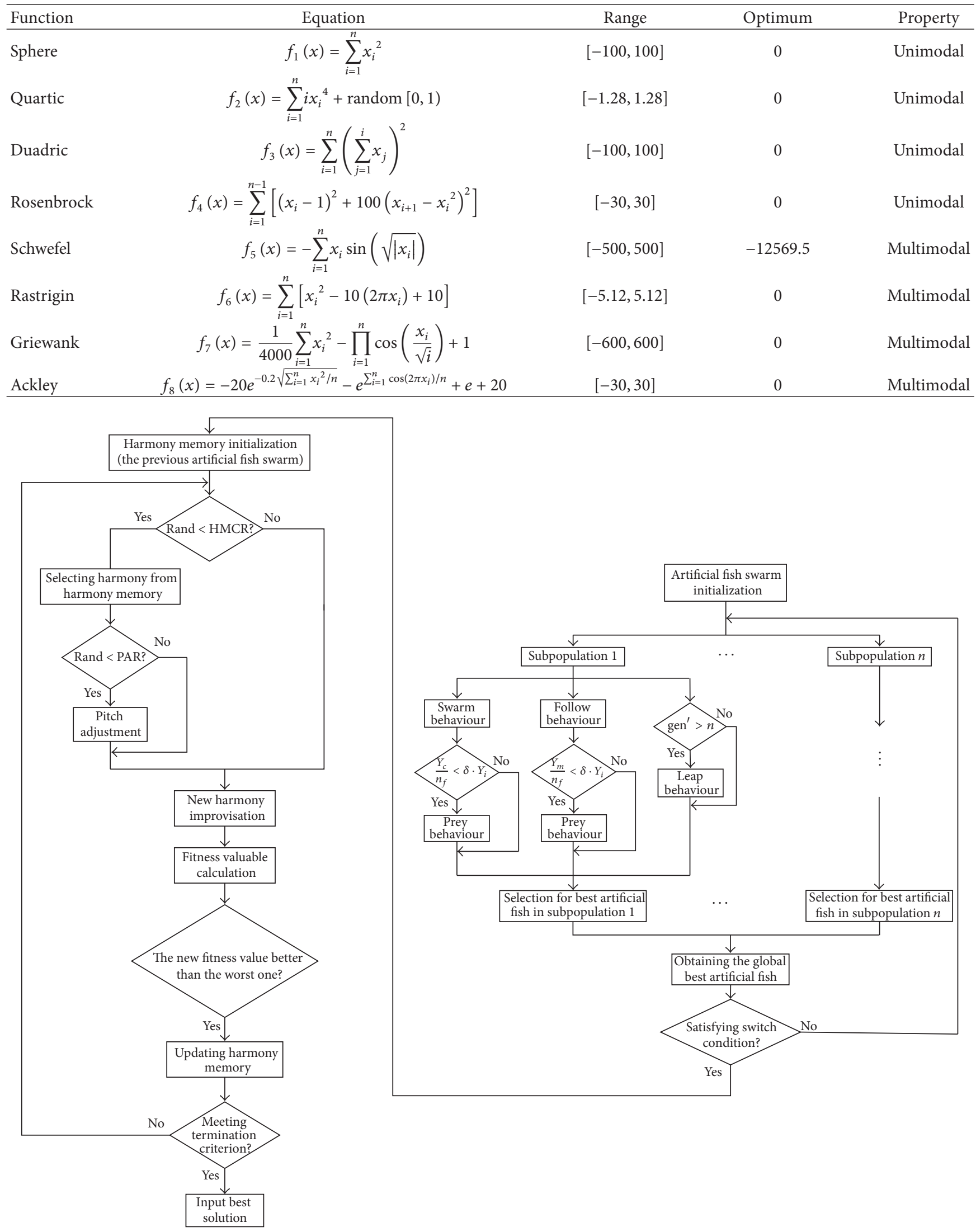

FIGURE 3: Flow for the proposed hybrid algorithm. 
TABLE 2: Comparison of results with other algorithms.

\begin{tabular}{|c|c|c|c|c|}
\hline & IAFSA-IHS & IBAFSA & IABHS & GDHS \\
\hline \multicolumn{5}{|l|}{$f_{1}$} \\
\hline Best & $3.0303 e-218$ & $3.4848 e-100$ & $3.8552 e-60$ & 0 \\
\hline Mean & $3.7514 e-220$ & $4.3574 e-99$ & $6.8125 e-59$ & 0 \\
\hline Worst & $9.3558 e-221$ & $6.1893 e-97$ & $7.2759 e-57$ & 0 \\
\hline Std.dev & $7.2377 e-218$ & $3.5238 e-99$ & $8.7153 e-59$ & 0 \\
\hline \multicolumn{5}{|l|}{$f_{2}$} \\
\hline Best & $4.3659 e-3$ & $5.7852 e-2$ & $1.5798 e-4$ & $8.9654 e-3$ \\
\hline Mean & $5.9283 e-2$ & $6.0237 e-2$ & $2.0721 e-2$ & $9.3571 e-2$ \\
\hline Worst & $1.1135 e-1$ & $1.3145 e-1$ & $5.3492 e-2$ & $7.5883 e-2$ \\
\hline Std.dev & $6.3844 e-2$ & $9.3561 e-2$ & $8.9443 e-3$ & $1.0184 e-2$ \\
\hline \multicolumn{5}{|l|}{$f_{3}$} \\
\hline Best & $6.901 e-3$ & $1.8312 e+3$ & $5.7163 e-4$ & $3.3694 e-2$ \\
\hline Mean & $5.4431 e-2$ & $7.5403 e+3$ & $6.7303 e-2$ & $5.2391 e-1$ \\
\hline Worst & $3.2967 e-1$ & $6.5727 e+4$ & $8.1835 e-2$ & $2.4749 e+0$ \\
\hline Std.dev & $2.3617 e-2$ & $5.4329 e+3$ & $4.3516 e-3$ & $4.7161 e-2$ \\
\hline \multicolumn{5}{|l|}{$f_{4}$} \\
\hline Best & $1.1364 e+1$ & $3.5801 e+1$ & $1.0075 e+1$ & $6.4625 e+1$ \\
\hline Mean & $9.5879 e+1$ & $6.0574 e+1$ & $9.5855 e+1$ & $8.5942 e+1$ \\
\hline Worst & $1.2841 e+2$ & $2.9714 e+1$ & $3.3575 e+1$ & $1.5399 e+2$ \\
\hline Std.dev & $9.9836 e+1$ & $3.4769 e+1$ & $1.4848 e+1$ & $7.6935 e+1$ \\
\hline \multicolumn{5}{|l|}{$f_{5}$} \\
\hline Best & $-1.1821 e+4$ & $-1.1108 e+4$ & $-1.1753 e+4$ & $-1.2024 e+4$ \\
\hline Mean & $-1.1028 e+4$ & $-9.9931 e+3$ & $-1.0387 e+4$ & $-1.1569 e+4$ \\
\hline Worst & $-1.0254 e+4$ & $-9.8301 e+3$ & $-9.4539 e+3$ & $-1.0965 e+4$ \\
\hline Std.dev & $-1.0879 e+4$ & $-1.0256 e+4$ & $-1.0024 e+4$ & $-1.2569 e+4$ \\
\hline \multicolumn{5}{|l|}{$f_{6}$} \\
\hline Best & 0 & $3.1827 e-4$ & $1.9847 e-3$ & $4.2084 e-4$ \\
\hline Mean & 0 & $9.5648 e-4$ & $9.0955 e-3$ & $1.5682 e-3$ \\
\hline Worst & 0 & $8.1815 e-3$ & $6.5276 e-1$ & $3.3524 e-2$ \\
\hline Std.dev & 0 & $3.1348 e-4$ & $6.4596 e-3$ & $1.2563 e-3$ \\
\hline \multicolumn{5}{|l|}{$f_{7}$} \\
\hline Best & 0 & $6.0477 e-10$ & $3.2291 e-14$ & $7.7708 e-16$ \\
\hline Mean & 0 & $7.5628 e-9$ & $9.2486 e-13$ & $6.2967 e-14$ \\
\hline Worst & 0 & $9.2466 e-8$ & $9.7123 e-12$ & $9.1222 e-13$ \\
\hline Std.dev & 0 & $1.3132 e-9$ & $2.2486 e-13$ & $1.5422 e-14$ \\
\hline \multicolumn{5}{|l|}{$f_{8}$} \\
\hline Best & $4.0107 e-16$ & $3.5687 e-15$ & $3.0026 e-13$ & $1.2627 e-15$ \\
\hline Mean & $7.5975 e-16$ & $7.0145 e-15$ & $5.2435 e-13$ & $3.3275 e-15$ \\
\hline Worst & $3.2354 e-15$ & $9.6461 e-14$ & $6.4564 e-12$ & $9.5619 e-15$ \\
\hline Std.dev & $5.5484 e-16$ & $5.5686 e-15$ & $1.3967 e-13$ & $2.0131 e-15$ \\
\hline
\end{tabular}




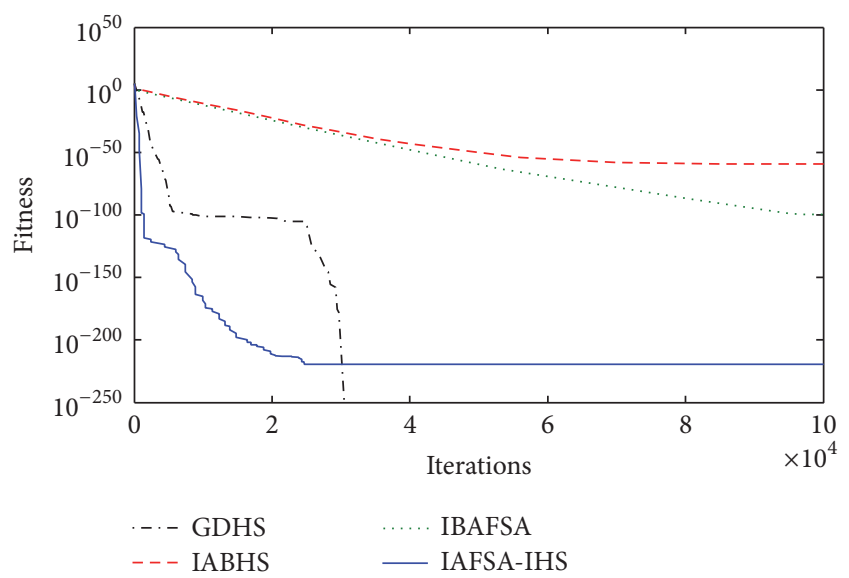

Figure 4: Sphere.

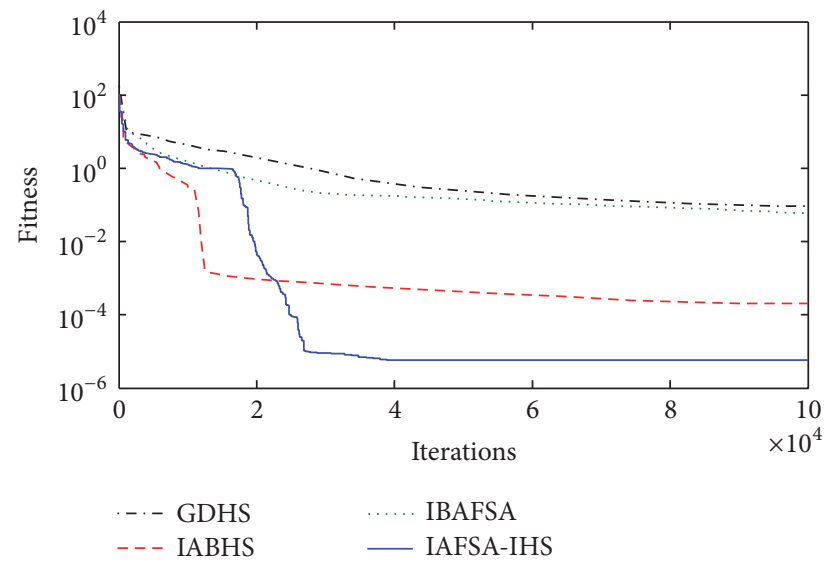

FIgURE 5: Quartic.

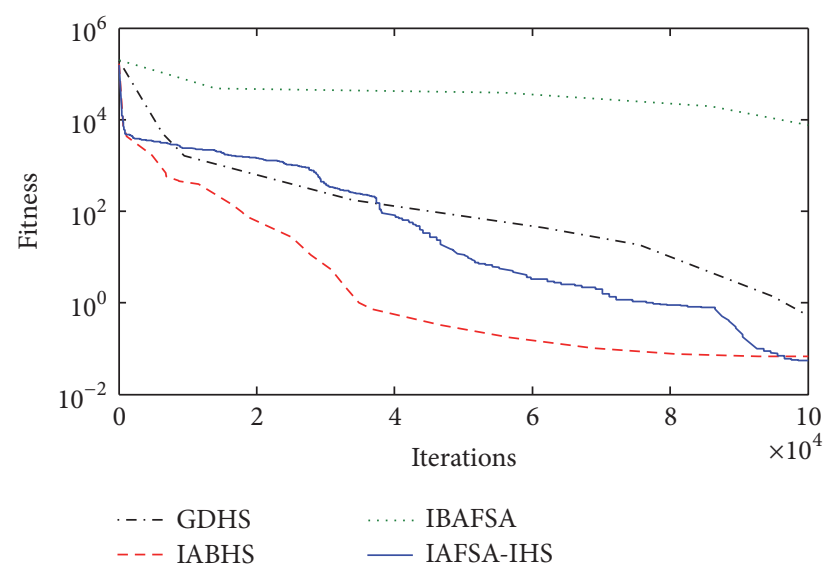

Figure 6: Duadric.

swarm regularly, and IABHS introduces the improvisation strategies with consideration of individual as well as dimension and self-adaptively adjusts HMCR, PAR, $b w$. All of those improvements are aimed at strengthening the convergence

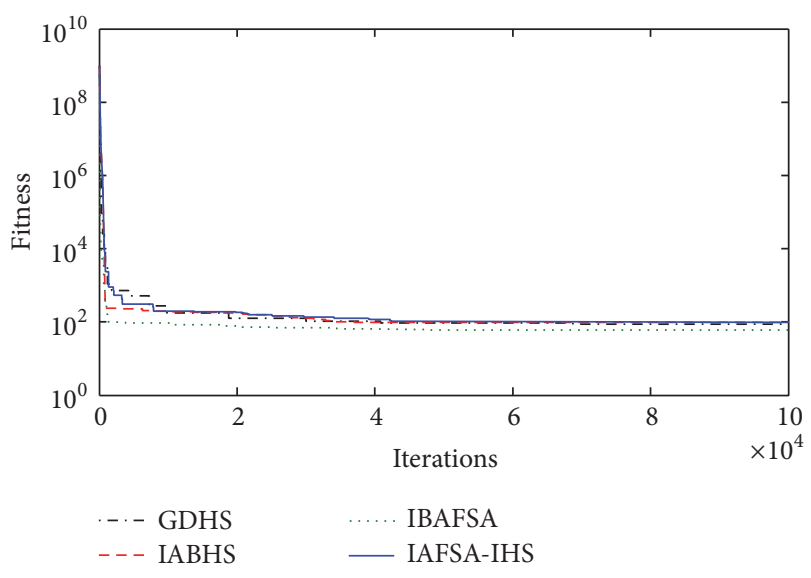

FIgURE 7: Rosenbrock.

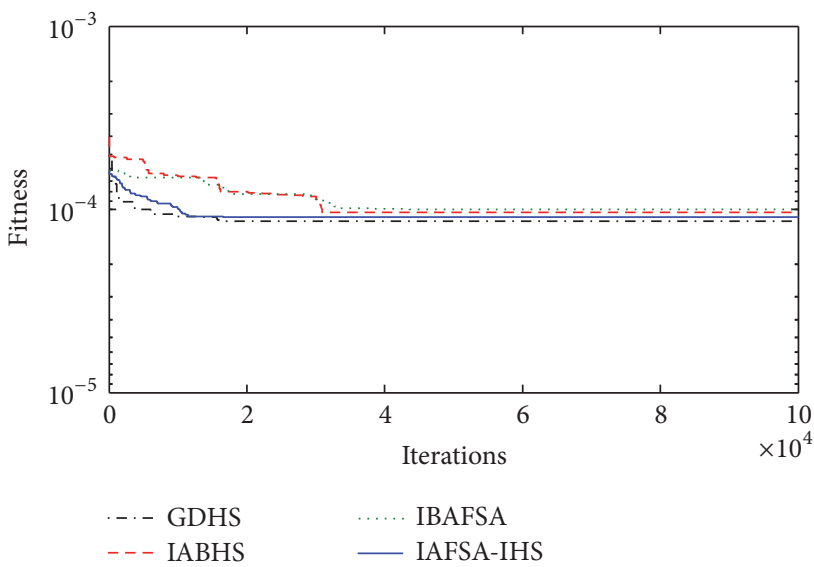

FIGURE 8: Schwefel.

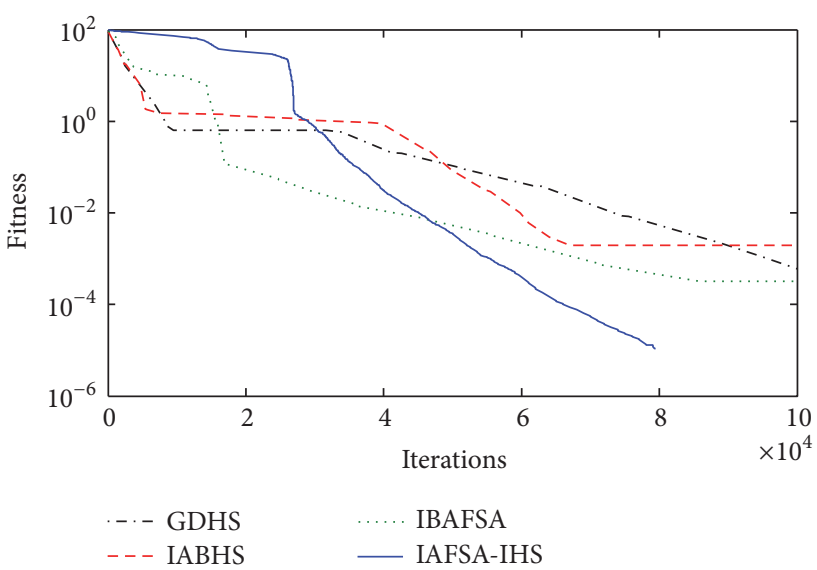

Figure 9: Rastrigin.

speed and accuracy, just as IAFSA-IHS does by means of the proposed modification in the paper. From the analysis of the result, these improvements indeed have effects as supposed. 


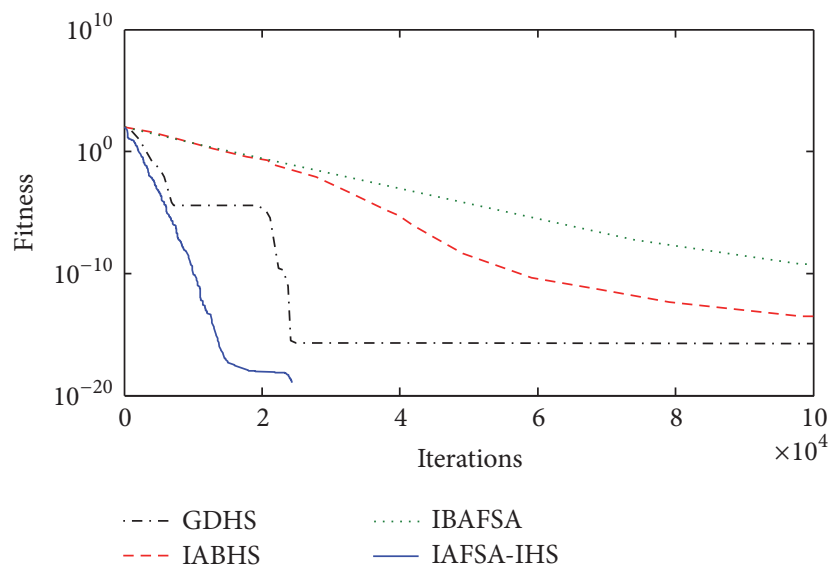

FIGURE 10: Griewank.

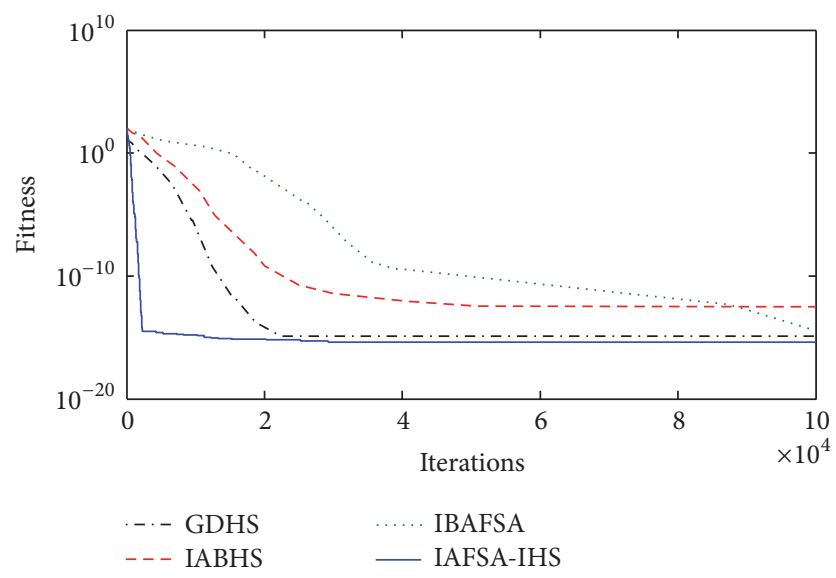

Figure 11: Ackley.

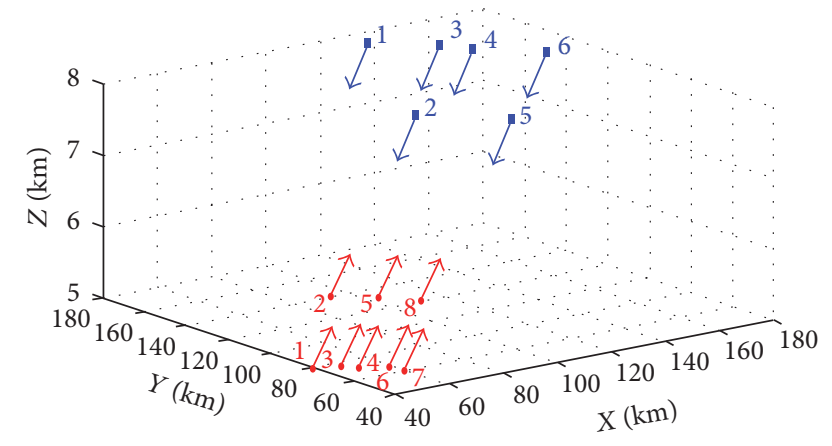

FIGURE 12: Air combat situation.

\subsection{Simulation of WTA Problems}

4.2.1. Example 1. There is red formation consisting of eight fighters confronting blue formation including six targets. Through calculation by (1) (8), situation information referring to $[17,33]$ on both sides is given in Table 3 and Figures $12-14$.

Descending velocity of each side with respect to the other side is obtained by (6) and (7) and shown in Tables 4 and 5.

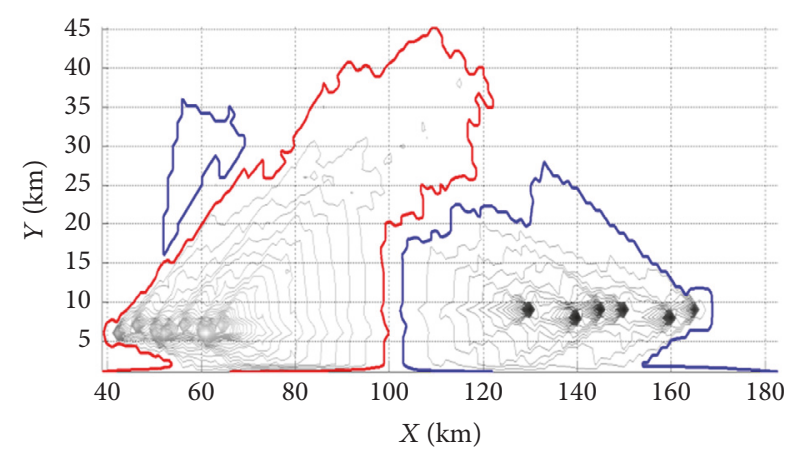

FIGURE 13: Distribution of combat power energy possessed by both formations in vertical plane.

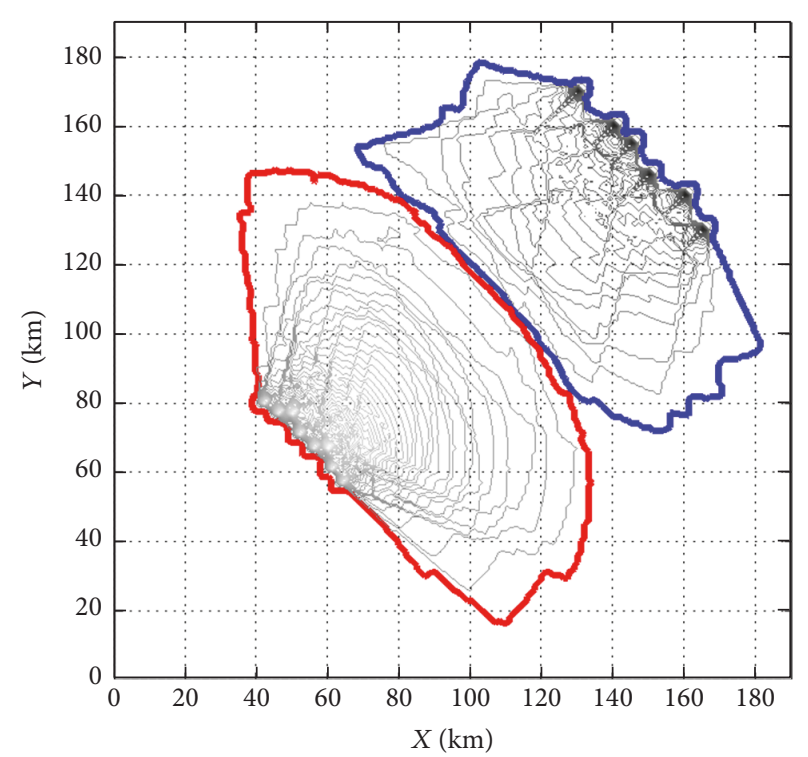

FIGURE 14: Distribution of combat power energy possessed by both formations in horizontal plane.

Relative superiority of red formation with regard to the blue one, $p_{i j}, 1 \leq i \leq 8,1 \leq j \leq 6$, is derived by (8) and is given in Table 6 .

$F_{i}, 1 \leq i \leq 8$, denotes fighter $i . T_{j}, 1 \leq j \leq 6$, is target $j$. ingen $=100, H_{0}=0.3, S=5$, visual $=8$, step $=4, c_{1}=$ $0.6, c_{2}=0.4, \varphi_{1}=0.6, \varphi_{2}=0.4$, and $\max$ gen $=200$; other parameters are set similarly as in verification tests.

Compared with three algorithms mentioned in verification tests, the optimization process containing four algorithms and the final assignment solution is shown in Figure 15 and Table 7.

It is obviously observed from Figure 15 and Table 7 that solution obtained by IAFSA-IHS gets the lowest fitness value but in slower convergence speed. By comparison, the results derived by GDHS, IBAFSA, and IABHS achieve higher fitness value. GDHS only acts better than IABHS, and the result got by IBAFSA is similar to the one got by IAFSA-IHS. It is concluded that the proposed IAFSA-IHS outperforms the others in solving the example. 
TABLE 3: Situation information on both sides.

\begin{tabular}{|c|c|c|c|c|c|c|c|c|c|c|}
\hline & $X / \mathrm{km}$ & $Y / \mathrm{km}$ & $Z / \mathrm{km}$ & $V x /(\mathrm{km} / \mathrm{h})$ & $V y /(\mathrm{km} / \mathrm{h})$ & $V z /(\mathrm{km} / \mathrm{h})$ & $C_{1}$ & $\mathrm{C}_{2}$ & $C_{3}$ & $\mathrm{C}_{4}$ \\
\hline \multicolumn{11}{|c|}{ Information on red formation } \\
\hline 1 & 40 & 79 & 5 & 662 & 662 & 0 & \multirow{8}{*}{361.65} & \multirow{8}{*}{43.59} & \multirow{8}{*}{1973.9} & \multirow{8}{*}{225.82} \\
\hline 2 & 44 & 76 & 6 & 662 & 662 & 0 & & & & \\
\hline 3 & 48 & 76 & 5 & 662 & 662 & 0 & & & & \\
\hline 4 & 50 & 70 & 5 & 662 & 662 & 0 & & & & \\
\hline 5 & 54 & 66 & 6 & 662 & 662 & 0 & & & & \\
\hline 6 & 58 & 66 & 5 & 662 & 662 & 0 & & & & \\
\hline 7 & 59 & 60 & 5 & 662 & 662 & 0 & & & & \\
\hline 8 & 62 & 56 & 6 & 662 & 662 & 0 & & & & \\
\hline \multicolumn{11}{|c|}{ Information on blue formation } \\
\hline 1 & 130 & 170 & 8 & -641 & -641 & -320 & \multirow{6}{*}{154.99} & \multirow{6}{*}{43.59} & \multirow{6}{*}{1789.7} & \multirow{6}{*}{451.64} \\
\hline 2 & 140 & 160 & 7 & -641 & -641 & -320 & & & & \\
\hline 3 & 145 & 155 & 8 & -641 & -641 & -320 & & & & \\
\hline 4 & 150 & 146 & 8 & -641 & -641 & -320 & & & & \\
\hline 5 & 160 & 140 & 7 & -641 & -641 & -320 & & & & \\
\hline 6 & 165 & 130 & 8 & -641 & -641 & -320 & & & & \\
\hline
\end{tabular}

TABLE 4: Descending velocity of red formation with regard to blue formation.

\begin{tabular}{lccccccrr}
\hline & F1 & F2 & F3 & F4 & F5 & F6 & F7 & F8 \\
\hline T1 & 0.0094 & 0.0094 & 0.0098 & 0.0094 & 0.0093 & 0.0095 & 0.0090 & 0.0088 \\
T2 & 0.0093 & 0.0094 & 0.0098 & 0.0095 & 0.0094 & 0.0098 & 0.0093 & 0.0091 \\
T3 & 0.0092 & 0.0093 & 0.0097 & 0.0094 & 0.0095 & 0.0098 & 0.0093 & 0.0092 \\
T4 & 0.0093 & 0.0095 & 0.0099 & 0.0097 & 0.0098 & 0.0102 & 0.0097 & 0.0096 \\
T5 & 0.0086 & 0.0088 & 0.0092 & 0.0091 & 0.0092 & 0.0096 & 0.0093 & 0.0092 \\
T6 & 0.0085 & 0.0088 & 0.0093 & 0.0091 & 0.0093 & 0.0098 & 0.0095 & 0.0095 \\
\hline
\end{tabular}

TABLE 5: Descending velocity of blue formation with regard to red formation.

\begin{tabular}{lccccccrr}
\hline & F1 & F2 & F3 & F4 & F5 & F6 & F7 & F8 \\
\hline T1 & 0.0078 & 0.0079 & 0.0082 & 0.0078 & 0.0077 & 0.0080 & 0.0075 & 0.0073 \\
T2 & 0.0077 & 0.0078 & 0.0082 & 0.0079 & 0.0079 & 0.0082 & 0.0077 & 0.0076 \\
T3 & 0.0076 & 0.0078 & 0.0081 & 0.0079 & 0.0079 & 0.0082 & 0.0078 & 0.0077 \\
T4 & 0.0077 & 0.0079 & 0.0083 & 0.0081 & 0.0081 & 0.0085 & 0.0081 & 0.0080 \\
T5 & 0.0071 & 0.0073 & 0.0077 & 0.0075 & 0.0077 & 0.0080 & 0.0077 & 0.0077 \\
T6 & 0.0071 & 0.0073 & 0.0077 & 0.0076 & 0.0078 & 0.0082 & 0.0079 & 0.0079 \\
\hline
\end{tabular}

TABLE 6: Relative superiority of red formation with regard to the blue one.

\begin{tabular}{|c|c|c|c|c|c|c|c|c|}
\hline & $\mathrm{F} 1$ & $\mathrm{~F} 2$ & F3 & F4 & F5 & F6 & F7 & F8 \\
\hline $\mathrm{T} 1$ & 0.5455 & 0.5454 & 0.5449 & 0.5455 & 0.5457 & 0.5452 & 0.5461 & 0.5464 \\
\hline $\mathrm{T} 2$ & 0.5456 & 0.5454 & 0.5448 & 0.5454 & 0.5454 & 0.5449 & 0.5456 & 0.5459 \\
\hline T3 & 0.5458 & 0.5455 & 0.5449 & 0.5454 & 0.5454 & 0.5448 & 0.5455 & 0.5457 \\
\hline $\mathrm{T} 4$ & 0.5457 & 0.5453 & 0.5447 & 0.5450 & 0.5449 & 0.5443 & 0.5450 & 0.5451 \\
\hline T5 & 0.5468 & 0.5464 & 0.5457 & 0.5460 & 0.5457 & 0.5451 & 0.5457 & 0.5457 \\
\hline T6 & 0.5469 & 0.5464 & 0.5457 & 0.5458 & 0.5455 & 0.5449 & 0.5453 & 0.5453 \\
\hline
\end{tabular}

TABLE 7: Results of four algorithms.

\begin{tabular}{llllllllll}
\hline & F1 & F2 & F3 & F4 & F5 & F6 & F7 & F8 & Fitness value \\
\hline GDHS & T6 & T1 & T3 & T4 & T5 & T2 & T4 & T1 & 2.2308 \\
IABHS & T1 & T1 & T6 & T4 & T5 & T3 & T4 & T2 & 2.2315 \\
IBAFSA & T6 & T3 & T5 & T2 & T1 & T2 & T1 & T4 & 2.2299 \\
IAFSA-IHS & T6 & T2 & T3 & T5 & T4 & T3 & T1 & T4 & 2.2298 \\
\hline
\end{tabular}


TABLE 8: Situation information on both sides.

\begin{tabular}{|c|c|c|c|c|c|c|c|c|c|}
\hline & $X / \mathrm{km}$ & $Y / \mathrm{km}$ & $Z / \mathrm{km}$ & $V x /(\mathrm{km} / \mathrm{h})$ & $V y /(\mathrm{km} / \mathrm{h})$ & $V z /(\mathrm{km} / \mathrm{h})$ & $C_{1}$ & $\mathrm{C}_{2}$ & $C_{3}$ \\
\hline \multicolumn{10}{|c|}{ Information on red formation } \\
\hline 1 & 50 & 80 & 5 & 828 & 552 & 138 & \multirow{8}{*}{361.65} & \multirow{8}{*}{43.59} & \multirow{8}{*}{1973.9} \\
\hline 2 & 51 & 76 & 6 & 828 & 552 & 138 & & & \\
\hline 3 & 52 & 72 & 5 & 828 & 552 & 138 & & & \\
\hline 4 & 53 & 69 & 6 & 828 & 552 & 138 & & & \\
\hline 5 & 56 & 65 & 6 & 552 & 828 & 138 & & & \\
\hline 6 & 60 & 64 & 5 & 552 & 828 & 138 & & & \\
\hline 7 & 63 & 63 & 6 & 552 & 828 & 138 & & & \\
\hline 8 & 68 & 62 & 5 & 552 & 828 & 138 & & & \\
\hline \multicolumn{10}{|c|}{ Information on blue formation } \\
\hline 1 & 155 & 143 & 7 & -402 & -768 & -283 & \multirow{6}{*}{154.99} & \multirow{6}{*}{43.59} & \multirow{6}{*}{1789.7} \\
\hline 2 & 150 & 144 & 7 & -402 & -768 & -283 & & & \\
\hline 3 & 140 & 146 & 8 & -402 & -768 & -283 & & & \\
\hline 4 & 135 & 150 & 7 & -768 & -402 & -283 & & & \\
\hline 5 & 132 & 160 & 8 & -768 & -402 & -283 & & & \\
\hline 6 & 130 & 170 & 8 & -768 & -402 & -283 & & & \\
\hline
\end{tabular}

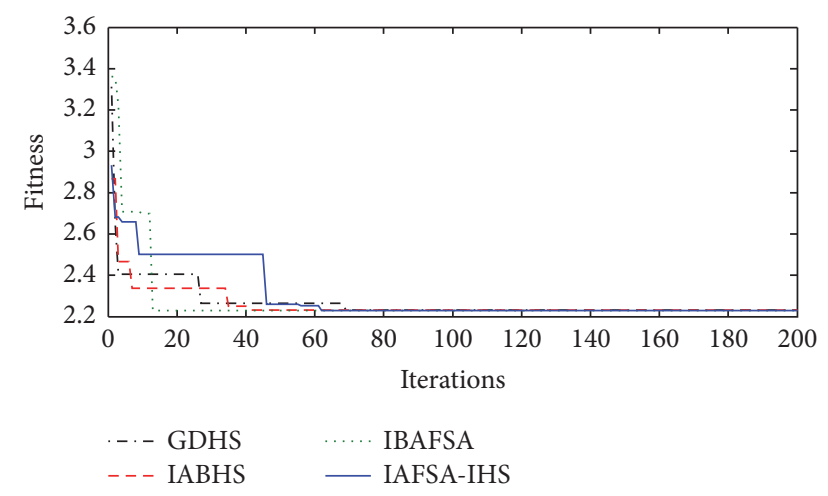

FIGURE 15: Optimization figure containing four algorithms.

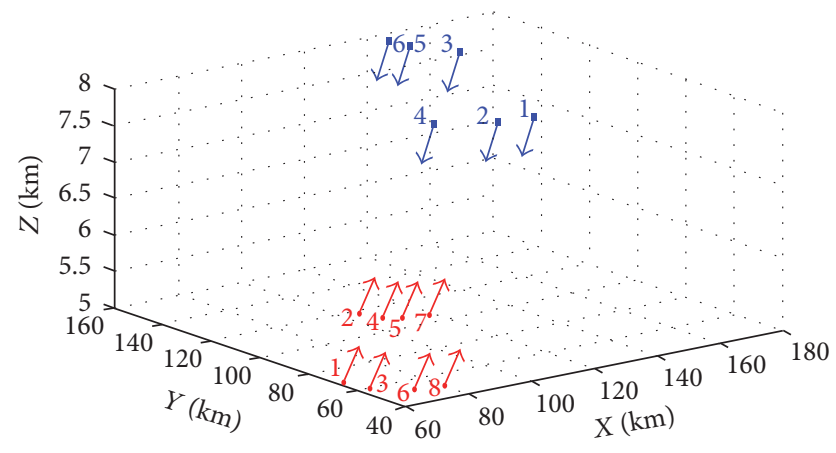

Figure 16: Air combat situation.

4.2.2. Example 2. To test its performance widely, another situation is chosen in simulation; the situation information is shown in Table 8 and Figures 16-18.

Descending velocity of each side with respect to the other side is gained by (6) and (7) and shown in Tables 9 and 10.

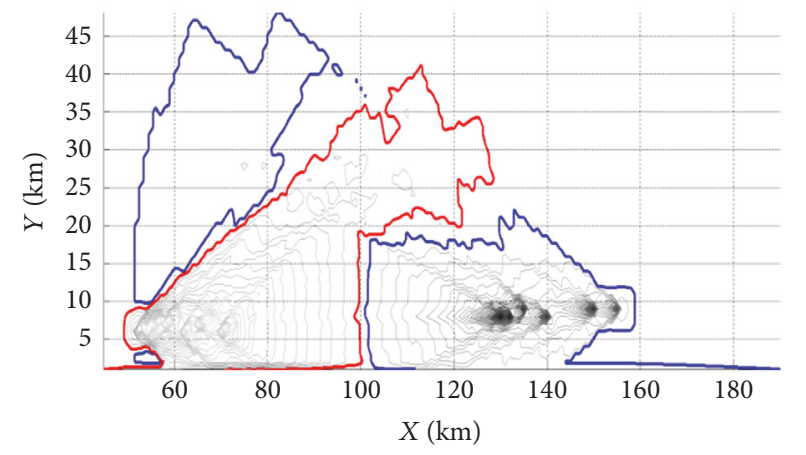

FIGURE 17: Distribution of combat power energy possessed by both formations in vertical plane.

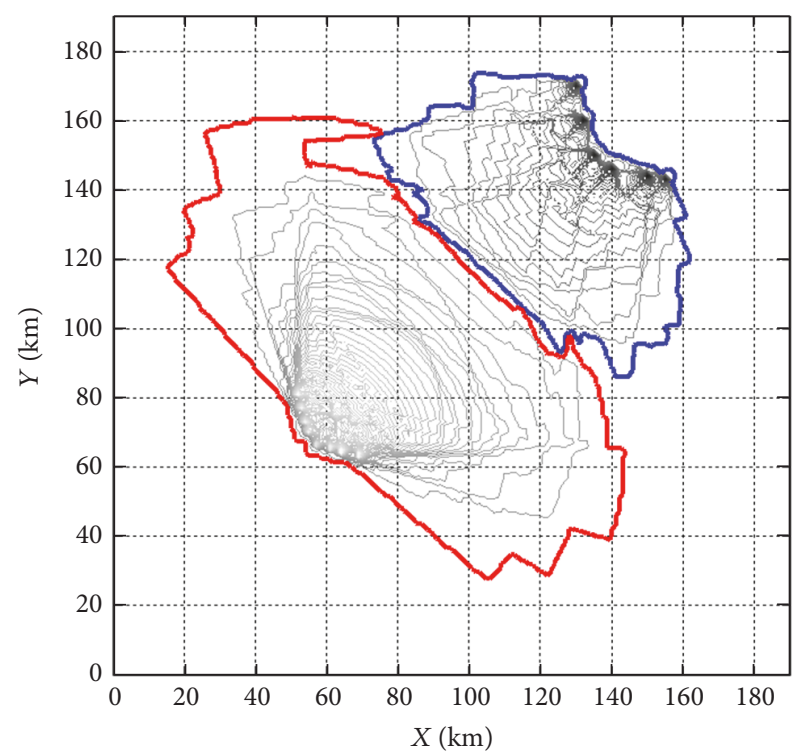

FIGURE 18: Distribution of combat power energy possessed by both formations in horizontal plane. 
TABLE 9: Descending velocity of red formation with regard to blue formation.

\begin{tabular}{lccccccrr}
\hline & F1 & F2 & F3 & F4 & F5 & F6 & F7 \\
\hline T1 & 0.0104 & 0.0100 & 0.0097 & 0.0095 & 0.0093 & 0.0095 & 0.0096 & 0.0098 \\
T2 & 0.0113 & 0.0110 & 0.0106 & 0.0104 & 0.0102 & 0.0104 & 0.0106 & 0.0109 \\
T3 & 0.0121 & 0.0117 & 0.0114 & 0.0112 & 0.0111 & 0.0114 & 0.0116 & 0.0120 \\
T4 & 0.0118 & 0.0115 & 0.0112 & 0.0110 & 0.0110 & 0.0113 & 0.0115 & 0.0120 \\
T5 & 0.0106 & 0.0104 & 0.0102 & 0.0101 & 0.0100 & 0.0104 & 0.0106 \\
T6 & 0.0101 & 0.0099 & 0.0097 & 0.0096 & 0.0096 & 0.0099 & 0.0102 & 0.0111 \\
\hline
\end{tabular}

TABLE 10: Descending velocity of blue formation with regard to red formation.

\begin{tabular}{lccccccrr}
\hline & F1 & F2 & F3 & F4 & F5 & F6 & F7 \\
\hline T1 & 0.0087 & 0.0084 & 0.0081 & 0.0079 & 0.0078 & 0.0079 & 0.0089 & 0.0082 \\
T2 & 0.0095 & 0.0092 & 0.0089 & 0.0087 & 0.0085 & 0.0088 & 0.0089 & 0.0092 \\
T3 & 0.0102 & 0.0099 & 0.0096 & 0.0094 & 0.0093 & 0.0096 & 0.0098 & 0.0101 \\
T4 & 0.0100 & 0.0097 & 0.0095 & 0.0093 & 0.0092 & 0.0095 & 0.0097 & 0.0101 \\
T5 & 0.0089 & 0.0087 & 0.0086 & 0.0084 & 0.0084 & 0.0087 & 0.0089 & 0.0093 \\
T6 & 0.0084 & 0.0083 & 0.0081 & 0.0080 & 0.0080 & 0.0083 & 0.0085 & 0.0089 \\
\hline
\end{tabular}

TABLE 11: Relative superiority of red formation with regard to the blue one.

\begin{tabular}{lccccccrr}
\hline & F1 & F2 & F3 & F4 & F5 & F6 & F7 \\
\hline T1 & 0.5440 & 0.5445 & 0.5450 & 0.5453 & 0.5456 & 0.5453 & 0.5452 & 0.5448 \\
T2 & 0.5428 & 0.5432 & 0.5437 & 0.5440 & 0.5442 & 0.5439 & 0.5437 \\
T3 & 0.5419 & 0.5423 & 0.5427 & 0.5429 & 0.5431 & 0.5427 & 0.5425 & 0.5420 \\
T4 & 0.5422 & 0.5425 & 0.5429 & 0.5431 & 0.5432 & 0.5428 & 0.5425 & 0.5420 \\
T5 & 0.5437 & 0.5439 & 0.5442 & 0.5444 & 0.5445 & 0.5440 & 0.5437 & 0.5431 \\
T6 & 0.5444 & 0.5447 & 0.5449 & 0.5451 & 0.5451 & 0.5446 & 0.5443 & 0.5437 \\
\hline
\end{tabular}

TABLE 12: Results of four algorithms.

\begin{tabular}{llllllllll}
\hline & F1 & F2 & F3 & F4 & F5 & F6 & F7 & F8 & Fitness value \\
\hline GDHS & T6 & T5 & T1 & T2 & T4 & T1 & 0 & T3 & 2.4894 \\
IABHS & T6 & T4 & T2 & T2 & T5 & T1 & T3 & T5 & 2.2415 \\
IBAFSA & T3 & T5 & T6 & T6 & T4 & T1 & T2 & T1 & 2.2413 \\
IAFSA-IHS & T6 & T5 & T4 & T6 & T2 & T1 & T3 & T5 & 2.2408 \\
\hline
\end{tabular}

Relative superiority of red formation with regard to the blue one, $p_{i j}, 1 \leq i \leq 8,1 \leq j \leq 6$, is derived by (8) and is given in Table 11.

Parameters remain unchanged as in Example 1, and the result can be seen in Figure 19 and Table 12.

From Figure 19 and Table 12, it is concluded that IAFSAIHS finds the best solution in highest convergence speed among the four algorithms. Besides, the initial solutions generated by IAFSA-IHS are far better than the ones of others. Among the other three algorithms, IBAFSA performs better in fitness value. IABHS presents a slightly inferior performance than the ones above. GDHS gets the worst results in fitness value as well as in convergence speed. From the example mentioned above, the proposed IAFSA-IHS

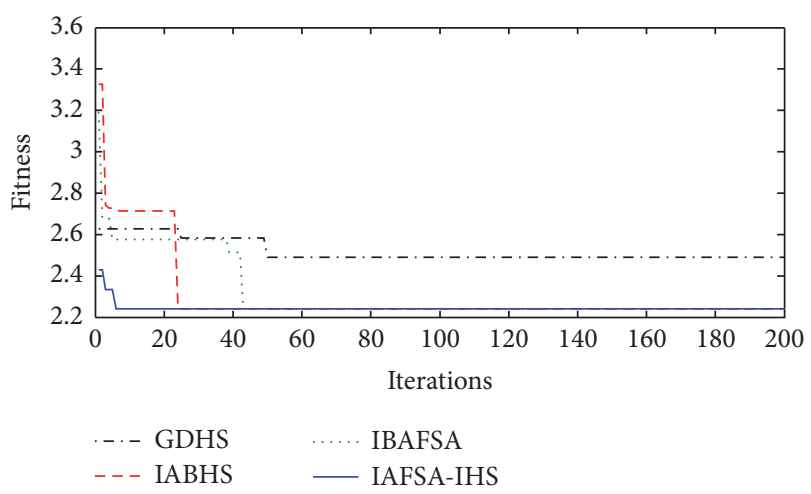

FIGURE 19: Optimization figure containing four algorithms. 
achieves a general better performance in both simulations that indeed demonstrates the effectiveness of IAFSA-IHS for WTA.

\section{Conclusion and Future Works}

A new approach to weapon-target assignment in cooperative air combat is proposed. Firstly, assignment model is constructed based on the concept of combat power potential. On the other hand, a hybrid algorithm combining improved AFSA with improved HS is proposed. Finally, the algorithm is tested by eight benchmark functions and two concrete WTA problems in air combat; the results validate the superiority of the proposed approach.

In the future, we will pay more attention to details about the combat power potential energy model of fighter in order to accurately reflect the practical cooperative air combat.

\section{Conflicts of Interest}

The authors declare that there are no conflicts of interest regarding the publication of this article.

\section{Acknowledgments}

This work was supported by the National Natural Science Foundation of China (61472441).

\section{References}

[1] O. Karasakal, "Air defense missile-target allocation models for a naval task group," Computers and Operations Research, vol. 35, no. 2, pp. 1759-1770, 2008.

[2] Z. R. Bogdanowicz, "A new efficient algorithm for optimal assignment of smart weapons to targets," Computers and Mathematics with Applications, vol. 58, no. 4, pp. 1965-1969, 2009.

[3] A. Mehmet, "Approximating the optimal mapping for weapon target assignment by fuzzy reasoning," Information Sciences, vol. 25, no. 5, pp. 30-44, 2014.

[4] Z. R. Bogdanowicz, "Advanced input generating algorithm for effect-based weapon-target pairing optimization," IEEE Transactions on Systems, Man, and Cybernetics Part A: Systems and Humans, vol. 42, no. 1, pp. 276-280, 2012.

[5] I. H. Toroslu and Y. Arslanoglu, "Genetic algorithm for the personnel assignment problem with multiple objectives," Information Sciences. An International Journal, vol. 177, no. 3, pp. 787803, 2007.

[6] Z.-J. Lee, S.-F. Su, and C.-Y. Lee, "Efficiently solving general weapon-target assignment problem by genetic algorithms with greedy eugenics," IEEE Transactions on Systems, Man, and Cybernetics, Part B: Cybernetics, vol. 33, no. 1, pp. 113-121, 2003.

[7] F. Bai, T. Q. Chang, and Y. Li, "An improved niche-based adaptive genetic algorithm for WTA problem solving," in Proceedings of the 2010 1st International Conference on Computational Problem-Solving, ICCP 2010, pp. 27-30, December 2010.

[8] A. R. McKendall Jr., J. Shang, and S. Kuppusamy, "Simulated annealing heuristics for the dynamic facility layout problem," Computers \& Operations Research, vol. 33, no. 8, pp. 2431-2444, 2006.
[9] X. Zeng, Y. Zhu, L. Nan, K. Hu, B. Niu, and X. He, "Solving weapon-target assignment problem using discrete particle swarm optimization," in Proceedings of the 6th World Congress on Intelligent Control and Automation, WCICA 2006, pp. 35623565, IEEE, Dalian, China, June 2006.

[10] R. Thangaraj, M. Pant, and A. K. Nagar, "Maximization of expected target damage value using quantum particle swarm optimization," in Proceedings of the International Conference on Developments in eSystems Engineering, DeSE 2009, pp. 329-334, IEEE, Abu Dhabi, UAE, December 2009.

[11] M.-C. Su, S.-C. Lai, S.-C. Lin, and L.-F. You, "A new approach to multi-aircraft air combat assignments," Swarm and Evolutionary Computation, vol. 6, pp. 39-46, 2012.

[12] Y. Li and Y. N. Dong, "Weapon-target assignment based on simulated annealing and discrete particle swarm optimization in cooperative air combat," Acta Aeronautica et Astronautica Sinica, vol. 31, no. 3, pp. 626-631, 2010.

[13] B. Xin, J. Chen, J. Zhang, L. Dou, and Z. Peng, "Efficient decision makings for dynamic weapon-target assignment by virtual permutation and tabu search heuristics," IEEE Transactions on Evolutionary Computation, vol. 40, no. 6, pp. 649-662, 2010.

[14] W. D. Song, C. W. Zhao, and J. X. Huo, "Improved differential evolution algorithm for solving WTA problem," Energy Procedia, vol. 11, pp. 1348-1353, 2011.

[15] J. Zhang, Z.-X. Wang, L. Chen, Z.-B. Wu, and J.-F. Lu, "Modeling and optimization on antiaircraft weapon-target assignment at multiple interception opportunity," Acta Armamentarii, vol. 35, no. 10, pp. 1644-1650, 2014.

[16] L. Chen, Z. X. Wang, Z. B. Wu et al., "A kind of antiaircraft weapon-target optimal assignment under earlier damage principle," Acta Aeronautica et Astronautica Sinica, vol. 35, no. 9, pp. 2574-2582, 2014.

[17] T. Zhou, B. Ren, and L. Yu, "Modeling of air combat analysis based on combat power field," Journal of System Simulation, vol. 20, no. 3, pp. 738-745, 2008.

[18] X. L. Li, Z. J. Shao, and J. X. Qian, "An optimizing method based on autonomous animals: fish swarm algorithm," Systems Engineering Theory and Practice, vol. 22, no. 11, pp. 32-38, 2002.

[19] D. Yazdani, H. Nabizadeh, E. Mohamadzadeh Kosari, and A. Nadjaran Toosi, "Color quantization using modified artificial fish swarm algorithm," Lecture Notes in Computer Science, vol. 7106, no. 1, pp. 382-391, 2011.

[20] P. Xue, "Adaptive hybrid artificial fish school algorithm for solving the real roots of polynomials," in Proceedings of the International Conference on Intelligent Computing (ICIC 2014), Intelligent Computing Theory, vol. 8588 of Lecture Notes in Computer Science, pp. 48-54, Springer.

[21] S. A. El-Said, "Image quantization using improved artificial fish swarm algorithm," Soft Computing-A Fusion of Foundations, Methodologies and Applications, vol. 19, no. 9, pp. 26672679, 2015.

[22] C.-R. Wang, C.-L. Zhou, and J.-W. Ma, "An improved artificial fish-swarm algorithm and its application in feed-forward neural networks," in Proceedings of the 4th International Conference on Machine Learning and Cybernetics, pp. 2890-2894, 2005.

[23] M. Jiang, Y. Wang, F. Rubio, and D. Yuan, "Spread spectrum code estimation by artificial fish swarm algorithm," in Proceedings of the IEEE International Symposium on Intelligent Signal Processing (WISP '07), pp. 1-6, October 2007.

[24] M. A. K. Azad, A. M. A. C. Rocha, and E. M. G. P. Fernandes, "Improved binary artificial fish swarm algorithm for the 0-1 
multidimensional knapsack problems," Swarm and Evolutionary Computation, vol. 14, pp. 66-75, 2014.

[25] Z. W. Geem, J. H. Kim, and G. V. Loganathan, "A new heuristic optimization algorithm: harmony search," Simulation, vol. 76, no. 2, pp. 60-68, 2001.

[26] Y. Z. Chang, Z. W. Li, Y. X. Kou et al., "Method for formation selection in air combat under uncertain information condition," Systems Engineering and Electronics, vol. 11, no. 38, pp. 25522560, 2016.

[27] B. Liu, L. Wang, Y.-H. Jin, F. Tang, and D.-X. Huang, "Improved particle swarm optimization combined with chaos," Chaos, Solitons and Fractals, vol. 25, no. 5, pp. 1261-1271, 2005.

[28] S. Rahnamayan, H. R. Tizhoosh, and M. M. A. Salama, "Opposition-based differential evolution," IEEE Transactions on Evolutionary Computation, vol. 12, no. 1, pp. 64-79, 2008.

[29] J.-S. Chun, M.-K. Kim, H.-K. Jung, and S.-K. Hong, "Shape optimization of electromagnetic devices using immune algorithm," IEEE Transactions on Magnetics, vol. 33, no. 2, pp. 1876-1879, 1997.

[30] L. Wang, R. Yang, Y. Xu, Q. Niu, P. M. Pardalos, and M. Fei, "An improved adaptive binary harmony search algorithm," Information Sciences. An International Journal, vol. 232, pp. 5887, 2013.

[31] R.-P. Li, H.-B. Ouyang, L.-Q. Gao, and D.-X. Zou, "Learned harmony search algorithm and its application to 0-1 knapsack problems," Control and Decision, vol. 28, no. 2, pp. 205-210, 2013.

[32] M. Khalili, R. Kharrat, K. Salahshoor, and M. H. Sefat, "Global dynamic harmony search algorithm: GDHS," Applied Mathematics and Computation, vol. 228, pp. 195-219, 2014.

[33] B. L. Zhu, R. C. Zhu, and X. F. Xiong, Battle Plane Effectiveness Evaluation, Aviation Industry Press, Beijing, China, 1993. 


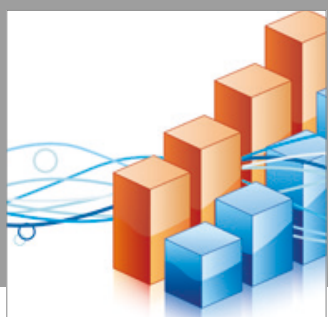

Advances in

Operations Research

vatersals

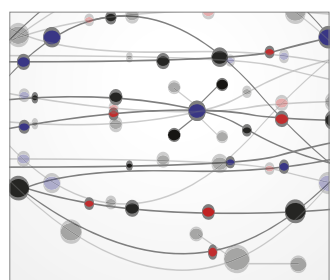

\section{The Scientific} World Journal
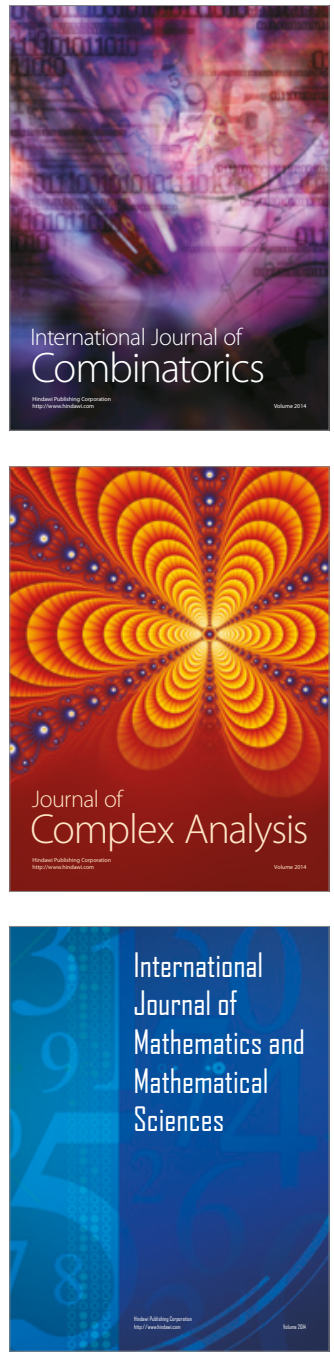
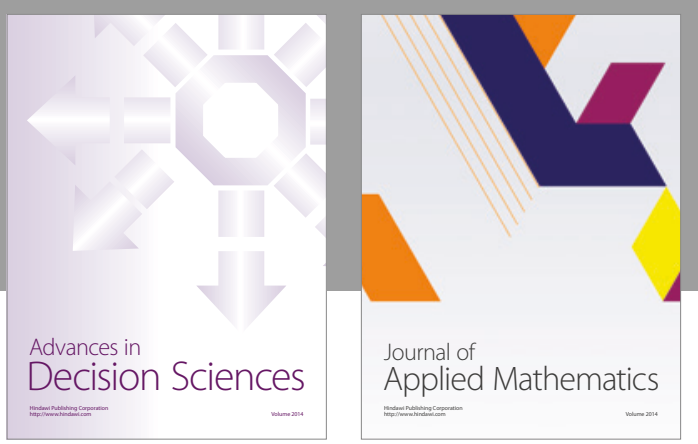

Algebra

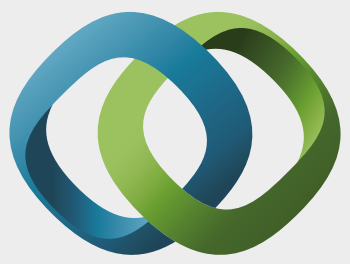

\section{Hindawi}

Submit your manuscripts at

https://www.hindawi.com
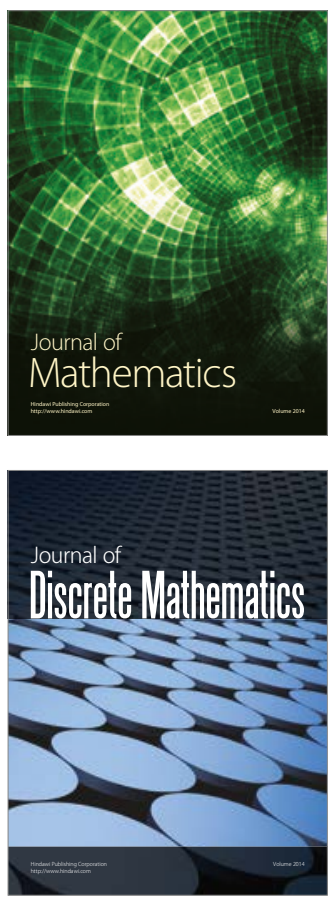

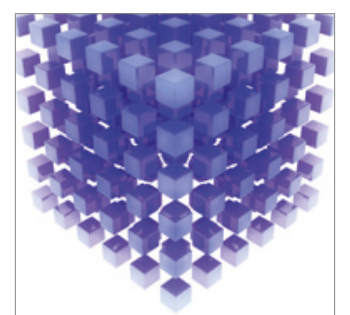

Mathematical Problems in Engineering
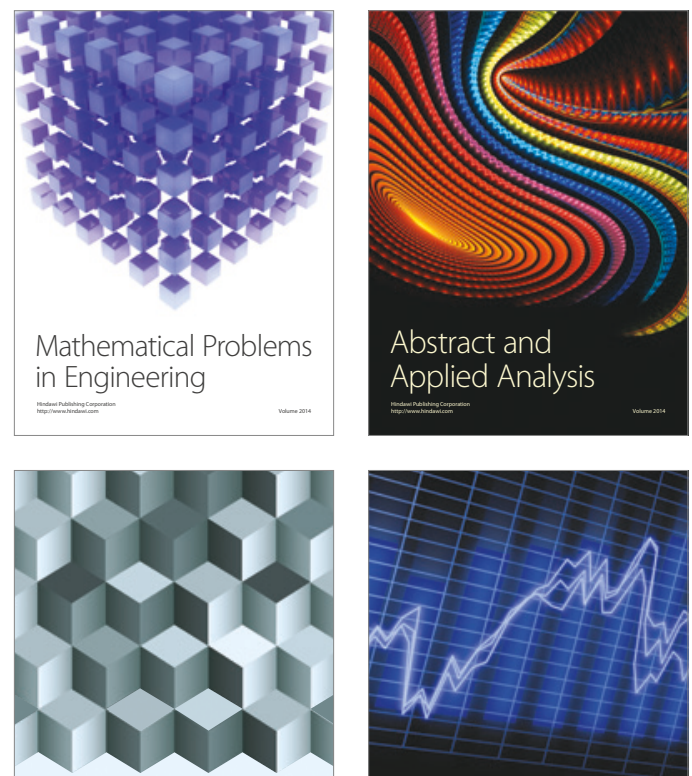

Journal of

Function Spaces

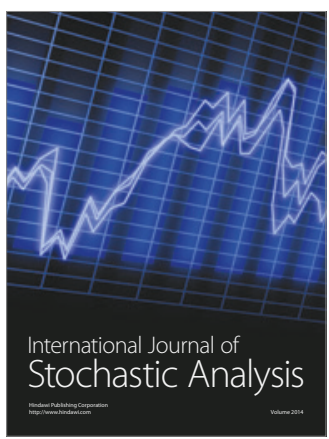

Probability and Statistics
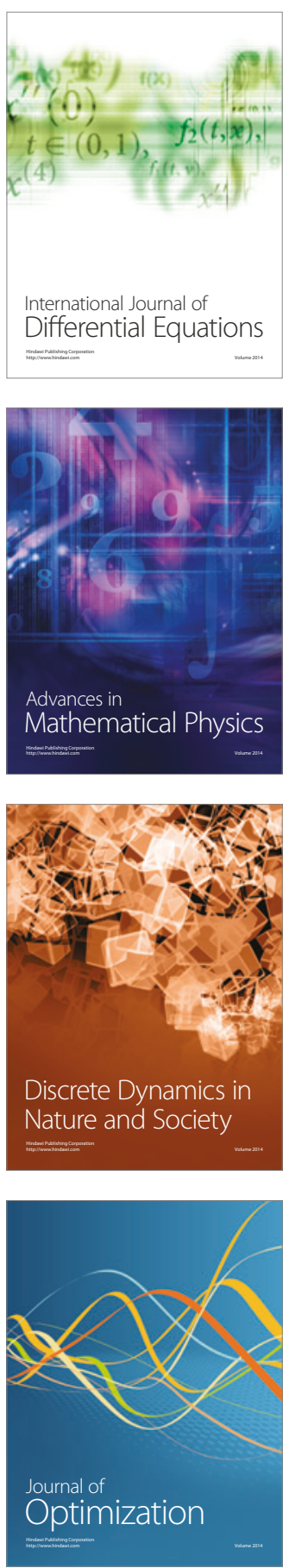